IASSNS-HEP-93/32

June 1993

\title{
Generalized quantum dynamics
}

\author{
Stephen L. Adler \\ Institute for Advanced Study \\ Princeton, NJ 08540
}

\begin{abstract}
We propose a generalization of Heisenberg picture quantum mechanics in which a Lagrangian and Hamiltonian dynamics is formulated directly for dynamical systems on a manifold with non-commuting coordinates, which act as operators on an underlying Hilbert space. This is accomplished by defining the Lagrangian and Hamiltonian as the real part of a graded total trace over the underlying Hilbert space, permitting a consistent definition of the first variational derivative with respect to a general operator-valued coordinate. The Hamiltonian form of the equations is expressed in terms of a generalized bracket operation, which is conjectured to obey a Jacobi identity. The formalism permits the natural implementation of gauge invariance under operator-valued gauge transformations. When an operator Hamiltonian exists as well as a total trace Hamiltonian, as is generally the case in complex quantum mechanics, one can make an operator gauge transformation from the Heisenberg to the Schrödinger picture. When applied to complex quantum mechanical systems with one bosonic or fermionic degree of freedom, the formalism gives the usual operator equations of motion, with the canonical commutation relations emerging as constraints associated with the operator gauge invariance. More generally, our methods permit the formulation of quaternionic quantum field theories with operator-valued gauge invariance, in which we conjecture that the operator constraints act as a generalization of the usual canonical commutators.
\end{abstract}




\section{Introduction}

The search for unification of the laws of physics has been closely intertwined with the discovery of wider classes of symmetries of the fundamental equations. Thus, the unification of electricity and magnetism in the Maxwell equations directly relates to both Abelian gauge invariance and relativistic kinematics; the advent of the standard model has as its underpinning the widening of the gauge principle from Abelian to non-Abelian groups. Current attempts at further unification are based largely on the use of supersymmetries relating bosonic to fermionic degrees of freedom.

In this paper we explore another possible direction for broadening the underlying symmetries, by generalizing from c-number to unitary operator gauge invariance of the fundamental equations. This approach grew out of an investigation [1] of quaternionic quantum mechanics and quaternionic quantum field theory, but the basic concepts can be understood without going through most of the details of quaternionic Hilbert space, and are presented here in a self-contained fashion. We begin, in Sec. 2, by developing an operator dynamics based on the idea of a total trace Lagrangian and Hamiltonian, and show how this can be used to formulate operator field equations which are covariant under operator-valued gauge transformations. We then apply the formalism in Sec. 3 to complex quantum mechanics, and show that it contains, and gener-

alizes, the usual canonical formalism. In Sec. 4, we explain a few simple facts about quaternionic Hilbert space, and then apply the total trace formalism to construct operator gauge invariant quaternionic field theories, the properties of which are discussed. Some brief concluding remarks are given in Sec. 5. Further details of the material presented in this paper can be found in the final two chapters of [1]. 


\section{Total trace Lagrangian formulation of quantum \\ dynamics and operator gauge invariance}

The primary tool for achieving invariance under operator-valued gauge transformations is the concept of a total trace Lagrangian, which will be developed in general form in this section. We begin by introducing an underlying complex or quaternionic Hilbert space, $V_{\mathbf{H}}$, which we assume to be the direct sum

$$
V_{\mathbf{H}}=V_{\mathbf{H}}^{+} \oplus V_{\mathbf{H}}^{-}
$$

of a Hilbert space $V_{\mathbf{H}}^{+}$of bosonic states and a Hilbert space $V_{\mathbf{H}}^{-}$of fermionic states. Following Witten [2] we define an operator $(-1)^{F}$ which counts fermion number modulo two, that is, $(-1)^{F}$ has eigenvalue +1 on all states in $V_{\mathbf{H}}^{+}$, and has eigenvalue -1 on all states in $V_{\mathbf{H}}^{-}$. Using this operator, we then define a trace operation $\operatorname{Tr} \mathcal{O}$ for a general operator $\mathcal{O}$ as follows,

$$
\operatorname{Tr} \mathcal{O}=\operatorname{Re} \operatorname{Tr}(-1)^{F} \mathcal{O}=\operatorname{Re} \sum_{n}\left\langle n\left|(-1)^{F} \mathcal{O}\right| n\right\rangle
$$

with Re the real part, with $\{|n\rangle\}$ any complete set of states, and with $\operatorname{Tr}$ the usual operator trace or diagonal sum. The operation Tr has the following useful properties:

(i) If $\mathcal{O}=\mathcal{O}^{-}$is fermionic, then $\operatorname{Tr} \mathcal{O}^{-}=0$, since

$$
\operatorname{Tr} \mathcal{O}^{-}=\operatorname{Re} \operatorname{Tr}\left[(-1)^{F} \mathcal{O}^{-}\right]=\operatorname{Re} \operatorname{Tr}\left[\mathcal{O}^{-}(-1)^{F}\right]=-\operatorname{Re} \operatorname{Tr}\left[(-1)^{F} \mathcal{O}^{-}\right]=-\operatorname{Tr} \mathcal{O}^{-}
$$

(ii) If $\mathcal{O}=\mathcal{O}^{+}$is bosonic, and $\mathcal{O}^{+}=\mathcal{O}_{(1)} \mathcal{O}_{(2)}$, then $\mathcal{O}_{(1)}$ and $\mathcal{O}_{(2)}$ are either both bosonic or both fermionic, and we have

$$
\begin{aligned}
\operatorname{Tr} \mathcal{O}_{(1)} \mathcal{O}_{(2)} & =\operatorname{Re} \operatorname{Tr}\left[(-1)^{F} \mathcal{O}_{(1)} \mathcal{O}_{(2)}\right]=\operatorname{Re} \operatorname{Tr}\left[\mathcal{O}_{(2)}(-1)^{F} \mathcal{O}_{(1)}\right] \\
& = \pm \operatorname{Re} \operatorname{Tr}\left[(-1)^{F} \mathcal{O}_{(2)} \mathcal{O}_{(1)}\right]= \pm \operatorname{Tr} \mathcal{O}_{(2)} \mathcal{O}_{(1)}
\end{aligned}
$$

with the + sign holding when $\mathcal{O}_{(1)}$ and $\mathcal{O}_{(2)}$ are both bosonic, and the - sign holding when $\mathcal{O}_{(1)}$ and $\mathcal{O}_{(2)}$ are both fermionic.

(iii) If $\mathcal{O}=-\mathcal{O}^{\dagger}$ is anti-self-adjoint, then

$$
\operatorname{Tr} \mathcal{O}=\operatorname{Re} \operatorname{Tr}\left[(-1)^{F} \mathcal{O}\right]=\operatorname{Re} \operatorname{Tr}\left[(-1)^{F} \mathcal{O}\right]^{\dagger}=\operatorname{Re} \operatorname{Tr}\left[(-1)^{F} \mathcal{O}^{\dagger}\right]=-\operatorname{Tr} \mathcal{O}
$$


and $\operatorname{Tr} \mathcal{O}$ vanishes. Correspondingly, if $\mathcal{O}$ is self-adjoint, then $\operatorname{Tr} \mathcal{O}$ agrees with $\operatorname{Tr}(-1)^{F} \mathcal{O}$, which is already real.

(iv) If $\operatorname{Tr} \sum_{r} \mathcal{O}_{r} \delta q_{r}=0$ for arbitrary independent operator variations $\delta q_{r}$, then each $\mathcal{O}_{r}$ must vanish, while if $\operatorname{Tr} \sum_{r} \mathcal{O}_{r} \delta q_{r}=0$ for operator variations $\delta q_{r}$ restricted to be of either bosonic or fermionic type, then the part of $\mathcal{O}_{r}$ of the same type must vanish. The first statement follows from

$$
\operatorname{Tr} \sum_{r} \mathcal{O}_{r} \delta q_{r}=\operatorname{Re}\left(\sum_{n, m, r}\left\langle n\left|(-1)^{F} \mathcal{O}_{r}\right| m\right\rangle\left\langle m\left|\delta q_{r}\right| n\right\rangle\right)
$$

choosing $\left\langle m\left|\delta q_{r}\right| n\right\rangle=\overline{\left\langle n\left|(-1)^{F} \mathcal{O}_{r}\right| m\right\rangle}$ (with the bar denoting complex or quaternion conjugation, the latter as defined in Sec. 4 below) gives

$$
\operatorname{Tr} \sum_{r} \mathcal{O}_{r} \delta q_{r}=\sum_{n, m, r}\left|\left\langle n\left|(-1)^{F} \mathcal{O}_{r}\right| m\right\rangle\right|^{2}
$$

which can vanish only if $(-1)^{F} \mathcal{O}_{r}=0$, which implies $\mathcal{O}_{r}=0$. The second statement follows by noting that when $\delta q_{r}$ is of bosonic or fermionic type, then property (i) implies that

$$
\operatorname{Tr} \sum_{r} \mathcal{O}_{r} \delta q_{r}=\operatorname{Tr} \sum_{r} \mathcal{O}_{r}^{(s)} \delta q_{r}=\operatorname{Tr} \sum_{r} \mathcal{O}_{r}^{(s)}\left[\delta q_{r}+\delta q_{r}^{(o)}\right]
$$

with $\mathcal{O}_{r}^{(s)}$ the part of $\mathcal{O}_{r}$ of the same type as $\delta q_{r}$ and $\delta q_{r}^{(o)}$ an arbitrary variation of the opposite type as $\delta q_{r}$. But $\delta q_{r}+\delta q_{r}^{(o)}$ is an unrestricted variation, so the first statement of property (iv) then implies $\mathcal{O}_{r}^{(s)}=0$

Let now $\left\{q_{r}(t)\right\}$ be a set of time-dependent quantum variables, which act as operators on the underlying Hilbert space, with each individual $q_{r}$ of either bosonic or fermionic type, and let $\left\{\dot{q}_{r}(t)\right\}$ be their time derivatives. We introduce an operator Lagrangian $L$ which is a polynomial function (or more generally, a Laurent series expandable function) of the variables $\left\{q_{r}\right\}$ and $\left\{\dot{q}_{r}\right\}$,

$$
L=L\left[\left\{q_{r}\right\},\left\{\dot{q}_{r}\right\}\right]
$$

and we define the total trace Lagrangian $\mathbf{L}$ by

$$
\mathbf{L}\left[\left\{q_{r}\right\},\left\{\dot{q}_{r}\right\}\right]=\operatorname{Tr} L\left[\left\{q_{r}\right\},\left\{\dot{q}_{r}\right\}\right],
$$


and the total trace action $\mathbf{S}$ by

$$
\mathbf{S}=\int_{-\infty}^{\infty} d t \mathbf{L}
$$

Because of property (i) of $\operatorname{Tr}$, any fermionic part of $L$ is automatically projected to zero, so there is no loss in generality in assuming that $L$ is bosonic. Similarly, by property (iii) of $\mathbf{T r}$, any anti-self-adjoint part of $L$ is automatically projected to zero, so we lose no generality by further specifying that $L$ is self-adjoint.

Let us now examine the consequences of requiring the total trace action to be stationary under arbitrary operator variations of the $\left\{q_{r}\right\}$, subject to the restriction that $\delta q_{r}$ be of the same bosonic or fermionic type as $q_{r}$. When we vary a given variable $q_{r}$, the variation of $L$ consists of a sum of terms of the form

$$
\mathcal{O}_{L} \delta q_{r} \mathcal{O}_{R}
$$

with $\mathcal{O}_{L, R}$ operators appearing respectively on the left $(L)$ and right $(R)$ of $\delta q_{r}$, which in general do not commute with $\delta q_{r}$. Inside the operation Tr, we can cyclically permute the factors in Eq. (3a) to get

$$
\operatorname{Tr} \mathcal{O}_{L} \delta q_{r} \mathcal{O}_{R}= \pm \operatorname{Tr} \mathcal{O}_{R} \mathcal{O}_{L} \delta q_{r}
$$

with the $+(-)$ sign corresponding, as in property (ii), to whether $\mathcal{O}_{R}$ is of bosonic (fermionic) type. Reordering all terms with the general form of Eq. (3a) this way, we are able to identify a well-defined operator $\delta \mathbf{L} / \delta q_{r}$, of the same bosonic or fermionic type as $q_{r}$, which obeys

$$
\delta \mathbf{L}=\operatorname{Tr} \frac{\delta \mathbf{L}}{\delta q_{r}} \delta q_{r}
$$

Similarly, varying one of the $\dot{q}_{r}$, we identify a well-defined operator $\delta \mathbf{L} / \delta \dot{q}_{r}$, again of the same type as $q_{r}$, which obeys

$$
\delta \mathbf{L}=\operatorname{Tr} \frac{\delta \mathbf{L}}{\delta \dot{q}_{r}} \delta \dot{q}_{r}
$$

When $q_{r}$ is of bosonic type, the order of the factors within $\operatorname{Tr}$ in Eqs. (3c,d) is irrelevant, but when $q_{r}$ is of fermionic type the factor ordering is significant, since by property (ii) of Eq. (1d), a minus sign appears when the order of two factors of fermionic type is reversed. In many applications, some of the $q_{r}$ are either self-adjoint or anti-self-adjoint in character. If $\delta q_{r}$ is further restricted to have the same adjointness 
character as $q_{r}$, then only the parts of $\delta \mathbf{L} / \delta q_{r}$ and $\delta \mathbf{L} / \delta \dot{q}_{r}$ which have the same (opposite) adjointness character as a bosonic (fermionic) $q_{r}$ are well-defined. It will be assumed henceforth that for those $q_{r}$ with definite adjointness character, the variational derivatives $\delta \mathbf{L} / \delta q_{r}$ and $\delta \mathbf{L} / \delta \dot{q}_{r}$ denote the operators of the same (opposite) adjointness character as a bosonic (fermionic) $q_{r}$ which obey Eqs. (3c,d). We note, finally, that the procedure just described cannot be extended to higher order variational derivatives. Since $\delta \mathbf{L} / \delta q_{r}$ is already an operator, a further variation will involve a sum of terms of the form of Eq. (3a), in which the $\delta q_{r}\left(\right.$ or $\left.\delta \dot{q}_{r}\right)$ factors are sandwiched between operators on left and right with which they do not commute. Without the trace, there is now no way to combine the terms in the sum into a single expression with infinitesimals on the right, and hence there is no definition of second variational derivatives analogous to Eqs. (3c,d).

Let us now impose an action principle, by requiring

$$
\delta \mathbf{S}=0
$$

under arbitrary same-type operator variations. Varying all the arguments $q_{r}$ and $\dot{q}_{r}$ of $\mathbf{L}$, we get

$$
\delta \mathbf{S}=\int_{-\infty}^{\infty} d t \delta \mathbf{L}=\int_{-\infty}^{\infty} d t \operatorname{Tr} \sum_{r}\left(\frac{\delta \mathbf{L}}{\delta q_{r}} \delta q_{r}+\frac{\delta \mathbf{L}}{\delta \dot{q}_{r}} \delta \dot{q}_{r}\right)
$$

which by an integration by parts becomes

$$
\delta \mathbf{S}=\left.\operatorname{Tr} \sum_{r} \frac{\delta \mathbf{L}}{\delta \dot{q}_{r}} \delta q_{r}\right|_{-\infty} ^{\infty}+\int_{-\infty}^{\infty} d t \operatorname{Tr} \sum_{r}\left[\frac{\delta \mathbf{L}}{\delta q_{r}}-\frac{d}{d t}\left(\frac{\delta \mathbf{L}}{\delta \dot{q}_{r}}\right)\right] \delta q_{r} .
$$

So if we take the variations $\delta q_{r}$ to vanish at $\pm \infty$, requiring $\delta \mathbf{S}=0$ gives, by property (iv), the operator equations of motion (the generalized Euler-Lagrange equations)

$$
\frac{\delta \mathbf{L}}{\delta q_{r}}-\frac{d}{d t}\left(\frac{\delta \mathbf{L}}{\delta \dot{q}_{r}}\right)=0
$$

Corresponding to the Lagrangian form of the equations in Eq. (4d), we can set up a Hamiltonian form by the usual method of making a Legendre transformation. Defining the momentum operator $p_{r}$ conjugate to $q_{r}$ by

$$
p_{r} \equiv \frac{\delta \mathbf{L}}{\delta \dot{q}_{r}}
$$


we define the total trace Hamiltonian $\mathbf{H}$ by

$$
\mathbf{H}=\operatorname{Tr} \sum_{r} p_{r} \dot{q}_{r}-\mathbf{L}
$$

We then have, under general same-type operator variations,

$$
\delta \mathbf{H}=\operatorname{Tr} \sum_{r}\left(\delta p_{r} \dot{q}_{r}+p_{r} \delta \dot{q}_{r}\right)-\operatorname{Tr} \sum_{r}\left(\frac{\delta \mathbf{L}}{\delta \dot{q}_{r}} \delta \dot{q}_{r}+\frac{\delta \mathbf{L}}{\delta q_{r}} \delta q_{r}\right)
$$

which substituting Eqs. (5a) and (4d), and using property (ii), becomes

$$
\delta \mathbf{H}=\operatorname{Tr} \sum_{r}\left( \pm \dot{q}_{r} \delta p_{r}-\dot{p}_{r} \delta q_{r}\right)
$$

with the $+(-)$ sign chosen according as whether $q_{r}$ is of bosonic (fermionic) type. Equation (5d) shows that $\mathbf{H}$ is a total trace functional of the operators $\left\{q_{r}\right\}$ and $\left\{p_{r}\right\}$,

$$
\mathbf{H}=\mathbf{H}\left[\left\{q_{r}\right\},\left\{p_{r}\right\}\right],
$$

with the operator variational derivatives

$$
\frac{\delta \mathbf{H}}{\delta q_{r}}=-\dot{p}_{r}, \quad \frac{\delta \mathbf{H}}{\delta p_{r}}= \pm \dot{q}_{r} .
$$

As in the case of the Lagrangian variations, when $q_{r}$ has a definite adjointness character, the variations $\delta \mathbf{H} / \delta q_{r}$ and $\delta \mathbf{H} / \delta p_{r}$ denote the operators obeying Eq. (5d) which have the same (opposite) adjointness character as a bosonic (fermionic) $q_{r}$. We note, finally, that with $p_{r}$ defined as in Eq. (5a), if the EulerLagrange equations are satisfied but arbitrary variations $\delta q_{r}$ are permitted at $t= \pm \infty$, then Eq. (4c) implies that

$$
\delta \mathbf{S}=\left.\operatorname{Tr} \sum_{r} p_{r} \delta q_{r}\right|_{-\infty} ^{\infty}
$$

This formula and the others involving $\mathbf{S}$ have obvious generalizations when the time interval $(-\infty, \infty)$ is replaced by $\left(T_{1}, T_{2}\right)$, for arbitrary finite $T_{1,2}$.

Continuing in analogy with the standard Hamiltonian formalism, let $\mathbf{A}\left[\left\{q_{r}\right\},\left\{p_{r}\right\}\right]$ and $\mathbf{B}\left[\left\{q_{r}\right\},\left\{p_{r}\right\}\right]$ be any two total trace functionals of the operator arguments $\left\{q_{r}\right\}$ and $\left\{p_{r}\right\}$, and let us define their generalized Poisson bracket

$$
\{\mathbf{A}, \mathbf{B}\} \equiv \operatorname{Tr} \sum_{r}( \pm)\left(\frac{\delta \mathbf{A}}{\delta q_{r}} \frac{\delta \mathbf{B}}{\delta p_{r}}-\frac{\delta \mathbf{B}}{\delta q_{r}} \frac{\delta \mathbf{A}}{\delta p_{r}}\right)
$$


with the $+(-)$ sign again corresponding to $q_{r}$ bosonic (fermionic). Then for a general total trace functional $\mathbf{A}\left[\left\{q_{r}\right\},\left\{p_{r}\right\}\right]$ we have

$$
\{\mathbf{A}, \mathbf{H}\}=\operatorname{Tr} \sum_{r}( \pm)\left(\frac{\delta \mathbf{A}}{\delta q_{r}} \frac{\delta \mathbf{H}}{\delta p_{r}}-\frac{\delta \mathbf{H}}{\delta q_{r}} \frac{\delta \mathbf{A}}{\delta p_{r}}\right)=\operatorname{Tr} \sum_{r}\left(\frac{\delta \mathbf{A}}{\delta q_{r}} \dot{q}_{r}+\frac{\delta \mathbf{A}}{\delta p_{r}} \dot{p}_{r}\right)=\frac{d}{d t} \mathbf{A}
$$

and since by construction the generalized Poisson bracket is anti-symmetric in its arguments,

$$
\{\mathbf{A}, \mathbf{B}\}=-\{\mathbf{B}, \mathbf{A}\}
$$

it follows that the time derivative of $\mathbf{H}$ vanishes,

$$
\frac{d}{d t} \mathbf{H}=\{\mathbf{H}, \mathbf{H}\}=0
$$

An important, and perhaps difficult, open question concerning the generalized Poisson bracket of Eq. (6a) is whether it satisfies a Jacobi identity. That is, let $\mathbf{A}\left[\left\{q_{r}\right\},\left\{p_{r}\right\}\right], \mathbf{B}\left[\left\{q_{r}\right\},\left\{p_{r}\right\}\right]$ and $\mathbf{C}\left[\left\{q_{r}\right\},\left\{p_{r}\right\}\right]$ be any three total trace functionals of the operator arguments $\left\{q_{r}\right\},\left\{p_{r}\right\}$, and let us define the bracket

$$
[\mathbf{A}, \mathbf{B}, \mathbf{C}]=\{\mathbf{A},\{\mathbf{B}, \mathbf{C}\}\}+\{\mathbf{C},\{\mathbf{A}, \mathbf{B}\}\}+\{\mathbf{B},\{\mathbf{C}, \mathbf{A}\}\}
$$

which is totally antisymmetric in $\mathbf{A}, \mathbf{B}$ and $\mathbf{C}$. On the basis of a number of examples, we conjecture that

$$
[\mathbf{A}, \mathbf{B}, \mathbf{C}] \equiv 0
$$

but we do not have a proof. It clearly is an important issue to find either a proof or a counter-example. If the Jacobi identity is satisfied, then using Eq. (6b), the bracket $\{\mathbf{A}, \mathbf{B}\}$ of any two constants of the motion $\mathbf{A}$ and $\mathbf{B}$ is itself a constant of the motion.

We now have all the ingredients needed to give a generalized version of Heisenberg picture quantum mechanics. States are described by fixed vectors $|b\rangle \in V_{\mathbf{H}}^{+}$and $|f\rangle \in V_{\mathbf{H}}^{-}$, and so the inner product geometry specified by the set of all inner products $\left\{\left\langle b \mid b^{\prime}\right\rangle\right\}$ and $\left\{\left\langle f \mid f^{\prime}\right\rangle\right\}$ is automatically time independent. The time dependence of the operators $\left\{q_{r}\right\}$ and $\left\{p_{r}\right\}$ is completely specified by Eq. (5f), giving these operators at all times once their form is specified at some initial time (say $t=0$ ). The most general observable $\mathcal{O}$ will be a self-adjoint polynomial function (or Laurent expandable function) of $\left\{q_{r}\right\},\left\{p_{r}\right\}$ and the time $t$,

$$
\mathcal{O}=\mathcal{O}\left[\left\{q_{r}\right\},\left\{p_{r}\right\}, t\right]
$$


and its time dependence is determined by using the Leibnitz product rule and Eq. (5f). The expectation of $\mathcal{O}$ in any state $|b\rangle$ or $|f\rangle$ can be rewritten as a total trace functional according to

$$
\begin{aligned}
\langle\mathcal{O}\rangle=\left\{\begin{array}{l}
\langle b|\mathcal{O}| b\rangle=\operatorname{Tr} P_{b} \mathcal{O} \\
\langle f|\mathcal{O}| f\rangle=-\operatorname{Tr} P_{f} \mathcal{O},
\end{array}\right. & P_{b}=|b\rangle\left\langle b\left|, \quad P_{f}=\right| f\right\rangle\langle f| .
\end{aligned}
$$

This permits us to apply Eq. (6b), as generalized to the case in which $\mathbf{A}$ has an explicit time dependence, giving

$$
\frac{d}{d t}\langle\mathcal{O}\rangle=\langle\partial \mathcal{O} / \partial t\rangle+\{\langle\mathcal{O}\rangle, \mathbf{H}\}
$$

Transition probabilities can also be reexpressed as total trace functionals,

$$
\left|\left\langle b \mid b^{\prime}\right\rangle\right|^{2}=\operatorname{Tr} P_{b} P_{b^{\prime}}=\operatorname{Tr} P_{b^{\prime}} P_{b}, \quad\left|\left\langle f \mid f^{\prime}\right\rangle\right|^{2}=-\operatorname{Tr} P_{f} P_{f^{\prime}}=-\operatorname{Tr} P_{f^{\prime}} P_{f}
$$

and are time independent by virtue of the time independence of the projectors $P_{b}, P_{f}, \ldots$.

After this rather lengthy excursion into total trace Lagrangians and Hamiltonians, we are ready to introduce the concept of operator gauge invariance. In its simplest form, an operator gauge transformation consists of a transformation on the operators $q_{r}$ of the form

$$
q_{r} \rightarrow U_{r} q_{r} U_{r}^{\dagger}+\Delta q_{r}\left[U_{r}\right]
$$

with each $U_{r}$ a unitary operator of bosonic type,

$$
U_{r} U_{r}^{\dagger}=U_{r}^{\dagger} U_{r}=1, \quad\left[(-1)^{F}, U_{r}\right]=0
$$

and with $\Delta q_{r}\left[U_{r}\right]$ an inhomogeneous term calculable in terms of the operator $U_{r}$. The Lagrangian $L$ of Eq. (2a) cannot in general be constructed to be invariant under the transformation of Eq. (8a), but we will find that we can readily construct Lagrangians $L$ in the form

$$
L=\sum_{r} L_{r}
$$

which transform under Eq. (8a) as

$$
L \rightarrow \sum_{r} U_{r} L_{r} U_{r}^{\dagger}
$$


As a consequence, although the operator Lagrangian $L$ is not invariant, the total trace Lagrangian

$$
\mathbf{L}=\operatorname{Tr} L
$$

is invariant under Eq. (8a),

$$
\mathbf{L} \rightarrow \mathbf{L}
$$

by virtue of the properties of $U_{r}$ in Eq. (8b) together with the cyclic invariance of the trace. We will also employ a second form of operator gauge transformation, in which the variables $q_{r}$ divide into three groups, the operators $q_{r}$ in the first group transforming as in Eq. (8a), those in the second group transforming as

$$
q_{r} \rightarrow U_{r}^{\prime} q_{r} U_{r}^{\prime \dagger}+\Delta q_{r}\left[U_{r}^{\prime}\right]
$$

and those in the third group transforming as

$$
q_{r} \rightarrow U_{r} q_{r} U_{r}^{\prime \dagger}
$$

with $U_{r}, U_{r}^{\prime}$ two independent unitary operators of bosonic type. We will now find Lagrangians $L$ in the form

$$
L=\sum_{r}\left(L_{r}+L_{r}^{\prime}\right)
$$

which transform under Eqs. (8a) and $(9 a, b)$ as

$$
L \rightarrow \sum_{r}\left(U_{r} L_{r} U_{r}^{\dagger}+U_{r}^{\prime} L_{r}^{\prime} U_{r}^{\prime \dagger}\right)
$$

Again, while the operator Lagrangian $L$ of Eq. (9c) is not operator gauge invariant, the corresponding total trace Lagrangian $\mathbf{L}$ is operator gauge invariant.

We now make a number of remarks concerning the structure and properties of operator gauge invariant total trace Lagrangians.

(1) We have written the equations of this section with $r$ a discrete index, but in many of the applications described in the next two sections, $r$ will be replaced by a continuum coordinate $\vec{x}$. 
(2) Varying Eqs. (8a), (9a) and (9b) with respect to $q_{r}$, the inhomogeneous term drops out, and we get respectively

$$
\begin{array}{ll}
\delta q_{r} \rightarrow U_{r} \delta q_{r} U_{r}^{\dagger} & \text { (first group) }, \\
\delta q_{r} \rightarrow U_{r}^{\prime} \delta q_{r} U_{r}^{\prime \dagger} & \text { (second group) }, \\
\delta q_{r} \rightarrow U_{r} \delta q_{r} U_{r}^{\prime \dagger} & \text { (third group) } .
\end{array}
$$

Hence when $\mathbf{L}$ is operator gauge invariant, the Eulerian derivative appearing in Eq. (4c) transforms as

$$
\begin{array}{ll}
E_{r} \rightarrow U_{r} E_{r} U_{r}^{\dagger} & \text { (first group) } \\
E_{r} \rightarrow U_{r}^{\prime} E_{r} U_{r}^{\prime \dagger} & \text { (second group) } \\
E_{r} \rightarrow U_{r}^{\prime} E_{r} U_{r}^{\dagger} & \text { (third group) } \\
E_{r} \equiv \frac{\delta \mathbf{L}}{\delta q_{r}}-\frac{d}{d t} \frac{\delta \mathbf{L}}{\delta \dot{q}_{r}}
\end{array}
$$

and the Euler-Lagrange equations $E_{r}=0$ are operator gauge covariant.

(3) A total trace version of the familiar Noether theorem of classical mechanics can be derived, as follows. Let $\delta \Lambda(t)$ be an infinitesimal operator parameterizing a set of operator variations $\delta q_{r}$ of the variables $q_{r}$, and let us assume that $\delta \mathbf{L}$ only involves $\delta \Lambda(t)$ and $\delta \dot{\Lambda}(t)$, but not $\delta \ddot{\Lambda}(t)$ or higher time derivatives (the Lagrangians studied in the next two sections all have this feature). Then we have

$$
\begin{aligned}
& \delta \mathbf{L}=\operatorname{Tr}\left(\frac{\delta \mathbf{L}}{\delta \Lambda} \delta \Lambda+\frac{\delta \mathbf{L}}{\delta \dot{\Lambda}} \delta \dot{\Lambda}\right) \\
& \delta \mathbf{S}=\left.\operatorname{Tr} \frac{\delta \mathbf{L}}{\delta \dot{\Lambda}} \delta \Lambda\right|_{-\infty} ^{\infty}+\int_{-\infty}^{\infty} d t \operatorname{Tr}\left(\left[\frac{\delta \mathbf{L}}{\delta \Lambda}-\frac{d}{d t}\left(\frac{\delta \mathbf{L}}{\delta \dot{\Lambda}}\right)\right] \delta \Lambda\right) .
\end{aligned}
$$

Assuming now that $\delta \Lambda$ vanishes rapidly enough at $t= \pm \infty$ so that all the $\delta q_{r}$ vanish there, and that the generalized Euler-Lagrange equations are satisfied for all times, then both $\delta \mathbf{S}$ and the surface terms in Eq. (10a) vanish, and independence of $\delta \Lambda(t)$ at different times implies

$$
0=\operatorname{Tr}\left(\left[\frac{\delta \mathbf{L}}{\delta \Lambda}-\frac{d}{d t}\left(\frac{\delta \mathbf{L}}{\delta \dot{\Lambda}}\right)\right] \delta \Lambda\right) .
$$

Suppose now that $\mathbf{L}$ is left invariant under the variations $\delta q_{r}$ parameterized by $\delta \Lambda$. Then $\delta \mathbf{L} / \delta \Lambda=0$, and Eq. (10b) simplifies to

$$
\operatorname{Tr}\left(\frac{d Q_{\Lambda}}{d t} \delta \Lambda\right)=0
$$


with $Q_{\Lambda}$ the "charge" defined by

$$
Q_{\Lambda}=\frac{\delta \mathbf{L}}{\delta \dot{\Lambda}}
$$

If $\mathbf{L}$ is invariant for arbitrary time-independent anti-self-adjoint operators $\delta \Lambda$, then Eq. (10c) implies the operator statement

$$
\frac{d Q_{\Lambda}}{d t}=0
$$

with $Q_{\Lambda}$ anti-self-adjoint. (Operator gauge transformations obey this condition trivially, with $Q_{\Lambda} \equiv 0$, since $\mathbf{L}$ is invariant for arbitrary time-dependent anti-self-adjoint $\delta \Lambda$.) On the other hand, in the case of Poincaré transformations, $\delta \Lambda$ is a $c$-number describing an infinitesimal translation or proper Lorentz transformation of the coordinates, and Eq. (10c) then only implies the total trace relation

$$
\frac{d}{d t} \operatorname{Tr} Q_{\Lambda}=0
$$

of which Eq. (6d) is a particular example.

An important special case is that in which $\delta \Lambda(t)$ parameterizes a linear transformation of the coordinates of the form

$$
\delta q_{r}=\delta \Lambda(t) \sum_{s} G_{r s} q_{s}
$$

with $G_{r s}$ independent of time and of the $q$ 's and $\dot{q}$ 's. Then we have

$$
\delta \dot{q}_{r}=\delta \dot{\Lambda}(t) \sum_{s} G_{r s} q_{s}+\delta \Lambda(t) \sum_{s} G_{r s} \dot{q}_{s}
$$

which together with Eq. (3d) implies that

$$
Q_{G}=\frac{\delta \mathbf{L}}{\delta \dot{\Lambda}}= \pm \sum_{r s} G_{r s} q_{s} \frac{\delta \mathbf{L}}{\delta \dot{q}_{r}}
$$

again with the $+(-)$ sign chosen according as whether $q_{r}$ is of bosonic (fermionic) type. Substituting Eq. (5a) into Eq. (11c) and taking the trace, we thus get

$$
\operatorname{Tr} Q_{G}=\operatorname{Tr}\left(\sum_{r s} p_{r} G_{r s} q_{s}\right)
$$


Let now $Q_{H}$ be the charge associated with a second linear transformation in which $G_{r s}$ is replaced by $H_{r s}$; then for the generalized Poisson bracket of $\operatorname{Tr} Q_{G}$ with $\operatorname{Tr} Q_{H}$, we find

$$
\begin{aligned}
\left\{\operatorname{Tr} Q_{G}, \operatorname{Tr} Q_{H}\right\} & =\operatorname{Tr}\left(\sum_{r s t}( \pm)\left(p_{r} G_{r s}( \pm) H_{s t} q_{t}-p_{r} H_{r s}( \pm) G_{s t} q_{t}\right)\right) \\
& =\operatorname{Tr}\left(\sum_{r t} p_{r} \sum_{s}\left(G_{r s} H_{s t}-H_{r s} G_{s t}\right) q_{t}\right)=\operatorname{Tr} Q_{[G, H]}
\end{aligned}
$$

Hence if a set of matrices $G, H, \ldots$ used to generate linear transformations of the $q$ 's obeys a Lie algebra, then the corresponding functionals $\operatorname{Tr} Q_{G}, \operatorname{Tr} Q_{H}, \ldots$ obey the same Lie algebra under the generalized Poisson bracket. This shows, for example, that in a Poincaré invariant theory defined by a total trace action, the total trace functionals defining the Poincaré generators will obey the Poincaré algebra under the bracket operation of Eq. (6a).

(4) When an operator gauge invariance is present, the problem of identifying physical observables becomes more subtle. Since observables should correspond to invariant geometric features of the quantum dynamics, only operator gauge invariant quantities can be observables. Thus, the expectation values of Eq. (7b), which are not operator gauge invariant for general states $|b\rangle,|f\rangle$, are in general not observables. One way to form observable quantities is to construct total trace functionals, similar to $\mathbf{L}$ but involving higher degree polynomials in the operators $q_{r}$, which are operator gauge invariant. Clearly, an infinite number of such observables can be constructed. A second way to form observable quantities is to focus on particular operators $\mathcal{O}_{r s}$ transforming under operator gauge transformations as

$$
\mathcal{O}_{r s} \rightarrow U_{r} \mathcal{O}_{r s} U_{s}^{\dagger}
$$

and on the co-transforming bases of states $\left|b_{r}^{(n)}\right\rangle,\left|b_{s}^{(n)}\right\rangle,\left|f_{r}^{(n)}\right\rangle,\left|f_{s}^{(n)}\right\rangle, n=1,2, \ldots$, which transform as

$$
\begin{aligned}
\left|b_{r}^{(n)}\right\rangle \rightarrow U_{r}\left|b_{r}^{(n)}\right\rangle, & \left|b_{s}^{(n)}\right\rangle \rightarrow U_{s}\left|b_{s}^{(n)}\right\rangle, \\
\left|f_{r}^{(n)}\right\rangle \rightarrow U_{r}\left|f_{r}^{(n)}\right\rangle, & \left|f_{s}^{(n)}\right\rangle \rightarrow U_{s}\left|f_{s}^{(n)}\right\rangle .
\end{aligned}
$$

Then the special class of matrix elements

$$
\left\langle b_{r}^{(n)}\left|\mathcal{O}_{r s}\right| b_{s}^{(m)}\right\rangle, \quad\left\langle f_{r}^{(n)}\left|\mathcal{O}_{r s}\right| f_{s}^{(m)}\right\rangle
$$


are operator gauge invariant, and hence observables.

(5) As we have seen, in theories constructed from a total trace Lagrangian, the Hamiltonian dynamics is governed by the operator variational equations of Eq. (5f), which are generated by the total trace Hamiltonian H. There appears to be no special reason for this dynamics to be unitary, that is, in general there is no reason to expect that there should be a unitary time evolution operator $U(t, 0)$, such that for all $q_{r}, p_{r}$ and all times $t$, the dynamics of Eq. (5f) is equivalent to

$$
q_{r}(t)=U^{\dagger}(t, 0) q_{r}(0) U(t, 0), \quad p_{r}(t)=U^{\dagger}(t, 0) p_{r}(0) U(t, 0)
$$

An equivalent statement in infinitesimal form is that in general there is no reason to expect that there should be an anti-self-adjoint operator Hamiltonian $\tilde{H}(t)$, such that for all $q_{r}, p_{r}$ and all times $t$, the dynamics of Eq. (5f) is equivalent to

$$
\dot{q}_{r}=\left[\tilde{H}(t), q_{r}\right], \quad \dot{p}_{r}=\left[\tilde{H}(t), p_{r}\right]
$$

It follows from these statements that total trace Lagrangian dynamics is, potentially, an even more general form of quantum mechanics than standard, operator Hamiltonian-based, quantum mechanics.

Suppose, however, that the dynamics is such that a $U(t, 0)$ and an $\tilde{H}(t)$ obeying Eqs. $(13 \mathrm{a}, \mathrm{b}) d o$ exist, for a theory with operator gauge invariance. Then we can make an operator gauge transformation

$$
q_{r}(t) \rightarrow U(t, 0) q_{r}(t) U^{\dagger}(t, 0) \equiv q_{r S}(t), \quad p_{r}(t) \rightarrow U(t, 0) p_{r}(t) U^{\dagger}(t, 0) \equiv p_{r S}(t)
$$

with a corresponding transformation for co-transforming states $|b\rangle,|f\rangle$,

$$
|b\rangle \rightarrow U(t, 0)|b\rangle \equiv\left|b_{S}(t)\right\rangle, \quad|f\rangle \rightarrow U(t, 0)|f\rangle \equiv\left|f_{S}(t)\right\rangle
$$

thus defining "Schrödinger picture" operators $q_{r S}(t), p_{r S}(t)$ and states $\left|b_{S}(t)\right\rangle$ and $\left|f_{S}(t)\right\rangle$. By construction, the operators $q_{r S}(t)$ and $p_{r S}(t)$ are time independent,

$$
q_{r S}(t)=q_{r S}(0), \quad p_{r S}(t)=p_{r S}(0)
$$

while the states $\left|b_{S}(t)\right\rangle$ and $\left|f_{S}(t)\right\rangle$ obey the Schrödinger time-development equation

$$
\frac{d}{d t}\left|b_{S}(t)\right\rangle=-\tilde{H}(t)\left|b_{S}(t)\right\rangle, \quad \frac{d}{d t}\left|f_{S}(t)\right\rangle=-\tilde{H}(t)\left|f_{S}(t)\right\rangle
$$


From this perspective, Schrödinger picture quantum mechanics appears as a rather special case of the more general quantum dynamics described by operator gauge invariant total trace Lagrangians.

It clearly is of great importance to determine under what circumstances the operator time development equations of Eq. (5f) are equivalent to a unitary evolution as in Eqs. (13a,b). It seems likely that this equivalence always holds in complex quantum mechanics, since there the standard canonical quantization rules give a constructive procedure for going from the Lagrangian $L$, interpreted now as a classical Lagrangian, to an operator Hamiltonian obeying Eq. (13b). In the case of quaternionic quantum mechanics the situation is far from clear, and we leave the problem of determining whether and when Eq. (5f) is equivalent to Eqs. (13a,b) as an important open question.

(6) As formulated up to this point, total trace quantum dynamics applies for arbitrary operator properties of the dynamical variables $\left\{q_{r}\right\},\left\{p_{r}\right\}$ at some initial time (say $t=0$ ), from which the Hamiltonian equations of motion can be integrated forwards to $t>0$ (and backwards to $t<0$ as well). Suppose now that for some subset of the variables $\left\{q_{R}\right\}$, the Lagrangian $\mathbf{L}$ is independent of the time derivatives $\left\{\dot{q}_{R}\right\}$. Then the corresponding canonical momenta $\left\{p_{R}\right\}$ vanish identically,

$$
p_{R}=\frac{\delta \mathbf{L}}{\delta \dot{q}_{R}} \equiv 0
$$

and the Euler-Lagrange equations for these variables degenerate to the constraints

$$
\frac{\delta \mathbf{L}}{\delta q_{R}}=0
$$

We are now dealing with a constrained Hamiltonian system, for which an operator generalization of the standard Dirac treatment of constrained systems will be needed. This may involve some subtleties, since the general Dirac prescription employs second variational derivatives of the Lagrangian, which, as discussed above, are not well-defined in the operator case. The situation in which some of the canonical momenta vanish identically is of course not the most general form of a constrained system, but it is precisely what occurs when a gauge invariance is present. We assume, in analogy with the standard Yang-Mills case, that the correct procedure for operator gauge invariant systems will be to adjoin to the operator constraints of Eqs. (14a,b) an equal number of operator gauge-fixing conditions, which break 
the operator gauge invariance. We conjecture that the constraints of Eqs. (14a,b), together with the operator gauge-fixing conditions, provide the minimum specification of operator properties of the $\left\{q_{r}\right\}$ and $\left\{p_{r}\right\}$ which are needed for a consistent theory. We will see, however, that there are examples in which it is possible to add further constraints beyond this minimum and still preserve consistency with the operator equations of motion. Finding a suitable operator generalization of the Dirac procedure, at least in the case of operator gauge invariant systems, and determining the precise conditions needed for operator specification, are again important open problems.

(7) Finally, some historical remarks. The concepts of operator-valued gauge transformations and a total trace action were introduced, without the $(-1)^{F}$ factor and real part of Eq. (1b), by Adler $[3,4,5]$ in the context of a theory termed "algebraic chromodynamics." The $(-1)^{F}$ factor was used by Witten [2] to define a topological index, which in our notation is simply $\operatorname{Tr} 1$. The necessity for including the real part in the definition of a trace for quaternionic Hilbert space was pointed out by Finkelstein, Jauch, and Speiser [6]. A general suggestion of operator-valued gauge transformations was also made by Mackey [7], with an implementation [8] which formulates the equivalence class, under unitary operator transformations, of complex Galilean invariant Hamiltonians. Since what we have done above is to set up a dynamics on a manifold with non-commuting coordinates $\left\{q_{r}\right\}$, the discussion of this and the subsequent two sections appears to be related to the non-commutative geometry program of A. Connes $[9,10]$. There are close analogies between the identification of observables in operator gauge invariant theories, and the identification of observables in general relativity and in conventional Yang-Mills gauge theories. Operators transforming as in Eq. (12a) are analogs of bitensor quantities in classical general relativity and of path ordered integrals in gauge theories. The suggestion that the generalization from special to general relativity should have an operator analog in the generalization from complex to quaternionic quantum mechanics, was first made by Finkelstein, Jauch, Schiminovich, and Speiser [11]. Because the analysis of this section has dealt with the general case, it has of necessity been rather abstract. Concrete illustrations of operator gauge invariant systems, in complex and quaternionic quantum mechanics and quantum field theory, are given in the next two sections. 


\section{Operator gauge invariant total trace Lagrangian formulation \\ of complex quantum mechanics}

We proceed in this section to illustrate the general formalism which we have just set up, in the familiar context of complex quantum mechanics and quantum field theory. In complex quantum mechanics, the left-acting operator $I$ defined by

$$
I=\sum_{n}|n\rangle i\langle n|
$$

commutes with all operators. (This permits the identification of $I$ with $i 1$, with 1 the unit operator and $i$ the right-acting imaginary unit, a notation which is standard in the complex quantum mechanics literature but which we shall not follow here. A discussion of right-acting versus left-acting algebras is given in Sec. 4 below.) Hence the complex specialization of operator gauge invariance consists in assuming that the operators $U_{r}, U_{r}^{\prime}$ of Eqs. (8a) and (9a,b) commute with $I$, which permits the inclusion of explicit factors of $I$ in the construction of operator gauge invariant total trace Lagrangians.

As our first example, we consider a single self-adjoint bosonic coordinate $q(t)$ obeying Galilean invariant dynamics. The conventional Lagrangian for this model (with the mass taken as unity for convenience) is

$$
L_{q}^{c}=\frac{1}{2} \dot{q}^{2}+\frac{1}{2}\{\dot{q}, A(q)\}_{+}-V(q)
$$

with $A(q)$ and $V(q)$ self-adjoint functions of $q$ and $\{,\}_{+}$the conventional anti-commutator; this Lagrangian clearly does not have any simple transformation properties under the operator transformation

$$
q \rightarrow U q U^{\dagger}, \quad U U^{\dagger}=U^{\dagger} U=1
$$

To achieve covariance, we follow the standard procedure of replacing the ordinary time derivative $\partial_{0}=\partial / \partial t$ by a covariant derivative $\hat{D}_{0}$, defined as

$$
\hat{D}_{0} q \equiv \frac{\partial q}{\partial t}+\left[B_{0}, q\right]
$$

with $B_{0}$ an anti-self-adjoint operator gauge potential. Under the transformation of Eq. (16b), $B_{0}$ is taken to transform as

$$
B_{0} \rightarrow U B_{0} U^{\dagger}-\frac{\partial U}{\partial t} U^{\dagger}=U B_{0} U^{\dagger}+U \frac{\partial}{\partial t} U^{\dagger}
$$


as a consequence of which $\hat{D}_{0} q$ transforms as

$$
\begin{aligned}
\hat{D}_{0} q \rightarrow & \frac{\partial}{\partial t}\left(U q U^{\dagger}\right)+\left(U B_{0} U^{\dagger}-\frac{\partial U}{\partial t} U^{\dagger}\right) U q U^{\dagger}-U q U^{\dagger}\left(U B_{0} U^{\dagger}+U \frac{\partial}{\partial t} U^{\dagger}\right) \\
& =U\left(\frac{\partial q}{\partial t}+\left[B_{0}, q\right]\right) U^{\dagger}=U\left(\hat{D}_{0} q\right) U^{\dagger} .
\end{aligned}
$$

Hence if we redefine the Lagrangian of Eq. (16a) as

$$
L_{q}=\frac{1}{2}\left(\hat{D}_{0} q\right)^{2}+\frac{1}{2}\left\{\hat{D}_{0} q, A(q)\right\}_{+}-V(q)
$$

then under operator gauge transformations $L_{q}$ transforms covariantly,

$$
L_{q} \rightarrow U L_{q} U^{\dagger}
$$

and the corresponding total trace Lagrangian $\mathbf{L}_{q}$ and action $\mathbf{S}_{q}$ defined by

$$
\mathbf{L}_{q}=\operatorname{Tr} L_{q}, \quad \mathbf{S}_{q}=\int_{-\infty}^{\infty} d t \mathbf{L}_{q}
$$

are invariant.

We have now achieved operator gauge invariance at the price of introducing an extra dynamical variable $B_{0}$. We must next investigate the structure of possible Lagrangians $\mathbf{L}_{B_{0}}$ to govern the dynamics of $B_{0}$. The usual Yang-Mills type field strength $F_{00}$ vanishes identically,

$$
F_{00}=\partial_{0} B_{0}-\partial_{0} B_{0}+\left[B_{0}, B_{0}\right]=0,
$$

and so cannot be used to construct a Lagrangian for $B_{0}$. However, there is one additional Lagrangian which can be formed from $B_{0}$,

$$
\mathbf{L}_{B_{0}}=\operatorname{Tr}\left(I B_{0}\right)
$$

with the inclusion of a factor of $I$ necessitated by the fact that $B_{0}$ is anti-self-adjoint. To see that Eq. (18b) defines a satisfactory Lagrangian, we note that it suffices to check its behavior under infinitesimal operator gauge transformations of the form

$$
U=1+\delta \Lambda, \quad \delta \Lambda=-\delta \Lambda^{\dagger}
$$


under which the first order variation of $B_{0}$ is

$$
\delta B_{0}=\left[\delta \Lambda, B_{0}\right]-\frac{\partial \delta \Lambda}{\partial t}=-\hat{D}_{0} \delta \Lambda
$$

From Eqs. (18b,d) we find

$$
\delta \mathbf{L}_{B_{0}}=\operatorname{Tr}\left(I \delta B_{0}\right)=\operatorname{Tr}\left(\left[B_{0}, I\right] \delta \Lambda\right)-\frac{\partial}{\partial t} \operatorname{Tr}(I \delta \Lambda)=-\frac{\partial}{\partial t} \operatorname{Tr}(I \delta \Lambda)
$$

which is a time derivative, and as a consequence the total trace action $\mathbf{S}_{B_{0}}$ defined by

$$
\mathbf{S}_{B_{0}}=\int_{-\infty}^{\infty} d t \mathbf{L}_{B_{0}}
$$

is invariant under Eq. (18c) when $\delta \Lambda$ vanishes at $t= \pm \infty$ [or more generally, when $\delta \Lambda(\infty)=\delta \Lambda(-\infty)$ ],

$$
\delta \mathbf{S}_{B_{0}}=0
$$

This argument of course does not imply that $\mathbf{S}_{B_{0}}$ is invariant under the global transformation of Eq. (16d), which can produce changes in the topological sector; rather, what we have shown is that $\mathbf{S}_{B_{0}}$ defines a form of topological action, which is constant within each distinct topological sector defined under operator valued gauge transformation.

An alternative argument for the invariance of $\mathbf{S}_{B_{0}}$ proceeds directly from Eq. (16d), which implies that

$$
\operatorname{Tr}\left(I B_{0}\right) \rightarrow \operatorname{Tr}\left[I\left(U B_{0} U^{\dagger}-\frac{\partial U}{\partial t} U^{\dagger}\right)\right]=\operatorname{Tr}\left(I B_{0}\right)-\operatorname{Tr}\left(I \frac{\partial U}{\partial t} U^{\dagger}\right)
$$

Writing $U=e^{\Lambda(t)}$, with $\Lambda=-\Lambda^{\dagger}$, and using the operator identity (which can be verified by power series expansion and term-by-term integration)

$$
\frac{\partial}{\partial t} e^{\Lambda}=\int_{0}^{1} d s e^{s \Lambda} \frac{\partial \Lambda}{\partial t} e^{(1-s) \Lambda}
$$

we have

$$
\operatorname{Tr}\left(I \frac{\partial U}{\partial t} U^{\dagger}\right)=\operatorname{Tr}\left(I \int_{0}^{1} d s e^{s \Lambda} \frac{\partial \Lambda}{\partial t} e^{(1-s) \Lambda} e^{-\Lambda}\right)=\operatorname{Tr}\left(I \int_{0}^{1} d s \frac{\partial \Lambda}{\partial t}\right)=\operatorname{Tr}\left(I \frac{\partial \Lambda}{\partial t}\right)
$$

Hence $\mathbf{S}_{B_{0}}$ is invariant whenever $\operatorname{Tr}[I \Lambda(\infty)]=\operatorname{Tr}[I \Lambda(-\infty)]$. This argument, as well as the infinitesimal one given above, extends immediately to the case in which $B_{0}$ is replaced by a space-time component of a four vector gauge potential and $\partial / \partial t$ is replaced by a space-time derivative. 
Let us now examine the dynamics following from a general linear combination of the actions $\mathbf{S}_{q}$ and $\mathbf{S}_{B_{0}}$. Forming the total Lagrangian $\mathbf{L}$ and action $\mathbf{S}$,

$$
\mathbf{L}=\mathbf{L}_{q}-\lambda_{0} \mathbf{L}_{B_{0}}, \quad \mathbf{S}=\mathbf{S}_{q}-\lambda_{0} \mathbf{S}_{B_{0}}
$$

with $\lambda_{0}$ a constant, and taking general operator variations, we get (with repeated use of the cyclic property of the trace)

$$
\begin{gathered}
\delta \mathbf{L}=\operatorname{Tr}\left\{\left[\hat{D}_{0} q+A(q)\right] \delta\left(\hat{D}_{0} q\right)+\left(\hat{D}_{0} q\right) \delta A(q)-\delta V(q)-\lambda_{0} I \delta B_{0}\right\} \\
=\operatorname{Tr}\left\{\left[\hat{D}_{0} q+A(q)\right]\left(\delta \dot{q}+\left[\delta B_{0}, q\right]+\left[B_{0}, \delta q\right]\right)+F\left(q, \hat{D}_{0} q\right) \delta q-\lambda_{0} I \delta B_{0}\right\} \\
=\operatorname{Tr}\left\{\left[\hat{D}_{0} q+A(q)\right] \delta \dot{q}+\left(\left[\hat{D}_{0} q+A(q), B_{0}\right]+F\left(q, \hat{D}_{0} q\right)\right) \delta q\right. \\
\left.+\left(\left[q, \hat{D}_{0} q+A(q)\right]-\lambda_{0} I\right) \delta B_{0}\right\}
\end{gathered}
$$

where we have defined a generalized force term $F\left(q, \hat{D}_{0} q\right)$ by

$$
\operatorname{Tr}\left[\left(\hat{D}_{0} q\right) \delta A(q)-\delta V(q)\right]=\operatorname{Tr}\left[F\left(q, \hat{D}_{0} q\right) \delta q\right]
$$

Hence

$$
\begin{aligned}
\frac{\delta \mathbf{L}}{\delta \dot{q}}=\hat{D}_{0} q+A(q), & \frac{\delta \mathbf{L}}{\delta q}=\left[\hat{D}_{0} q+A(q), B_{0}\right]+F\left(q, \hat{D}_{0} q\right), \\
\frac{\delta \mathbf{L}}{\delta \dot{B}_{0}}=0, \quad \frac{\delta \mathbf{L}}{\delta B_{0}} & =\left[q, \hat{D}_{0} q+A(q)\right]-\lambda_{0} I,
\end{aligned}
$$

and so the Euler-Lagrange equations following from $\delta \mathbf{S}=0$ consist of a dynamical equation for $q(t)$,

$$
\hat{D}_{0}\left[\hat{D}_{0} q+A(q)\right]=\frac{d}{d t}\left[\hat{D}_{0} q+A(q)\right]+\left[B_{0}, \hat{D}_{0} q+A(q)\right]=F\left(q, \hat{D}_{0} q\right)
$$

together with a constraint

$$
\left[q, \hat{D}_{0} q+A(q)\right]=\lambda_{0} I
$$

both of which are covariant under the operator gauge transformations of Eqs. (16b-e). When we rewrite the dynamics in total trace Hamiltonian form, we identify the canonical momentum conjugate to $q$ as

$$
p=\frac{\delta \mathbf{L}}{\delta \dot{q}}=\hat{D}_{0} q+A(q)
$$


and so the constraint of Eq. (20g) reads

$$
[q, p]=\lambda_{0} I
$$

and is just the standard canonical commutator with the identification $\lambda_{0}=\hbar(=1$ in microscopic units). Note that because of the real part in the definition of $\operatorname{Tr}$, we have $\operatorname{Tr} I=0$, and so taking $\operatorname{Tr}$ of the leftand right-hand sides of Eq. (21b) gives a consistent equation for non-zero $\lambda_{0}$,

$$
0=\operatorname{Tr}[q, p]=\lambda_{0} \operatorname{Tr} I=\lambda_{0} 0 .
$$

Carrying out the Legendre transform of Eq. (5b) we get

$$
\begin{aligned}
\mathbf{H} & =\operatorname{Tr}\left(\frac{\delta \mathbf{L}}{\delta \dot{q}} \dot{q}\right)-\mathbf{L} \\
& =\operatorname{Tr}\left\{\left[\hat{D}_{0} q+A(q)\right]\left(\hat{D}_{0} q-\left[B_{0}, q\right]\right)-\frac{1}{2}\left(\hat{D}_{0} q\right)^{2}-\left(\hat{D}_{0} q\right) A(q)+V(q)+\lambda_{0} I B_{0}\right\} \\
& =\operatorname{Tr}\left\{\frac{1}{2}\left(\hat{D}_{0} q\right)^{2}+V(q)+\left(\lambda_{0} I-\left[q, \hat{D}_{0} q+A(q)\right]\right) B_{0}\right\} \\
& =\operatorname{Tr}\left\{\frac{1}{2}[p-A(q)]^{2}+V(q)+\left(\lambda_{0} I-[q, p]\right) B_{0}\right\},
\end{aligned}
$$

which in the "Hamiltonian gauge"

$$
B_{0}=0
$$

simplifies to

$$
\mathbf{H}=\operatorname{Tr}\left\{\frac{1}{2}[p-A(q)]^{2}+V(q)\right\}
$$

Taking the operator variation of Eq. (22a), and recalling the definition of Eq. (20c), we get

$$
\delta \mathbf{H}=\operatorname{Tr}\{[p-A(q)][\delta p-\delta A(q)]+\delta V(q)\}=\operatorname{Tr}\{[p-A(q)] \delta p-F(q, p-A(q)) \delta q\} .
$$

Hence the total trace Hamiltonian equations of motion are

$$
\dot{q}=\frac{\delta \mathbf{H}}{\delta p}=p-A(q), \quad \dot{p}=-\frac{\delta \mathbf{H}}{\delta q}=F(q, p-A(q))
$$

which agree with Eqs. (16c), (20f) and (21a) when these are specialized to the gauge $B_{0}=0$.

We see, then, that the Heisenberg picture equations of motion and the canonical commutation relation for a Galilean particle both emerge from the operator gauge invariant, total trace Lagrangian formalism. The 
derivations just given are expressed in operator terms throughout; at no point did we introduce a classical Lagrangian and its "quantization." In complex quantum mechanics, the conventional canonical quantization route is of course still valid, and implies that there is an operator Hamiltonian $H$ given by the Weyl ordering of the corresponding classical Hamiltonian. For the model under study, we have

$$
H=\left\{\frac{1}{2}[p-A(q)]^{2}+V(q)\right\}_{W}
$$

where the Weyl ordering subscript $W$ implies symmetrization of $p$ with respect to the factors of $q$ in each term of $A(q)$, e.g.,

$$
\left\{p q^{n}\right\}_{W}=\left\{q^{n} p\right\}_{W}=\frac{1}{n+1}\left(q^{n} p+q^{n-1} p q+q^{n-2} p q^{2}+\ldots+q^{2} p q^{n-2}+q p q^{n-1}+p q^{n}\right) .
$$

With $[q, p]=\lambda_{0} I$, we then find that

$$
\frac{1}{I \lambda_{0}}[q, H]=p-A(q), \quad \frac{1}{-I \lambda_{0}}[p, H]=-F(q, p-A(q))
$$

since, as may be verified by some algebra, the Weyl ordering of $H$ leads to the same factor ordering in the force term $F$ as is obtained, via the cyclic property of the trace, from the operator variational definition of $F$ in Eq. (20c). Hence the equations of motion of Eq. (22c) are equivalent to

$$
\dot{q}=\lambda_{0}^{-1}[I H, q], \quad \dot{p}=\lambda_{0}^{-1}[I H, p],
$$

and so in the terminology of the preceding section, the dynamics is unitary. This permits us to transform from the Heisenberg picture to the Schrödinger picture, in which the operators are time independent and the co-transforming states carry the quantum dynamics.

In the example just given, all operators are bosonic, and so the $(-1)^{F}$ factor in the definition of $\operatorname{Tr}$ does not come into play. As our second example, we consider a single non-interacting fermion degree of freedom with mass $m$, described by the conventional Lagrangian

$$
L_{\psi}^{c}=\frac{I}{2}\left(\psi^{\dagger} \dot{\psi}-\dot{\psi}^{\dagger} \psi\right)-m \psi^{\dagger} \psi
$$

The Lagrangian of Eq. (24a) again does not transform simply under the operator transformation

$$
\psi \rightarrow U \psi U^{\dagger}, \quad U U^{\dagger}=U^{\dagger} U=1
$$


but as in the bosonic example, we can achieve covariance by replacing the time derivative by the covariant derivative $\hat{D}_{0}$,

$$
\hat{D}_{0} \psi \equiv \frac{\partial \psi}{\partial t}+\left[B_{0}, \psi\right], \quad\left(\hat{D}_{0} \psi\right)^{\dagger}=\hat{D}_{0} \psi^{\dagger}=\frac{\partial \psi^{\dagger}}{\partial t}+\left[B_{0}, \psi^{\dagger}\right]
$$

With $B_{0}$ transforming as in Eq. (16d), $\hat{D}_{0} \psi$ transforms as

$$
\hat{D}_{0} \psi \rightarrow U\left(\hat{D}_{0} \psi\right) U^{\dagger}, \quad \hat{D}_{0} \psi^{\dagger} \rightarrow U\left(\hat{D}_{0} \psi^{\dagger}\right) U^{\dagger},
$$

and so the redefined Lagrangian

$$
L_{\psi}=\frac{I}{2}\left[\psi^{\dagger} \hat{D}_{0} \psi-\left(\hat{D}_{0} \psi^{\dagger}\right) \psi\right]-m \psi^{\dagger} \psi
$$

transforms covariantly,

$$
L_{\psi} \rightarrow U L_{\psi} U^{\dagger}
$$

The corresponding total trace Lagrangian $\mathbf{L}_{\psi}$ and action $\mathbf{S}_{\psi}$,

$$
\mathbf{L}_{\psi}=\operatorname{Tr} L_{\psi}, \quad \mathbf{S}_{\psi}=\int_{-\infty}^{\infty} d t \mathbf{L}_{\psi}
$$

are then invariant.

Proceeding as in our first example, we examine the dynamics following from a general linear combination of the actions $\mathbf{S}_{\psi}$ and $\mathbf{S}_{B_{0}}$. Writing

$$
\mathbf{L}=\mathbf{L}_{\psi}-\lambda_{0} \mathbf{L}_{B_{0}}, \quad \mathbf{S}=\mathbf{S}_{\psi}-\lambda_{0} \mathbf{S}_{B_{0}}
$$

and taking general operator variations of $\psi$ and $B_{0}$, with $\delta \psi^{\dagger}=(\delta \psi)^{\dagger}$, we get

$$
\begin{aligned}
\delta \mathbf{L}= & \operatorname{Tr}\left\{\frac{I}{2}\left[\delta \psi^{\dagger} \hat{D}_{0} \psi+\psi^{\dagger} \delta\left(\hat{D}_{0} \psi\right)-\delta\left(\hat{D}_{0} \psi^{\dagger}\right) \psi-\left(\hat{D}_{0} \psi^{\dagger}\right) \delta \psi\right]\right. \\
& \left.-m\left(\delta \psi^{\dagger} \psi+\psi^{\dagger} \delta \psi\right)-\lambda_{0} I \delta B_{0}\right\} \\
= & \operatorname{Tr}\left(2(\delta \psi)^{\dagger}\left(I \hat{D}_{0} \psi-m \psi\right)-I\left[\left\{\psi, \psi^{\dagger}\right\}_{+}+\lambda_{0}\right] \delta B_{0}\right)+\frac{\partial}{\partial t} \operatorname{Tr}\left(I \psi^{\dagger} \delta \psi\right)
\end{aligned}
$$

We have here explicitly used properties (ii) and (iii) of $\operatorname{Tr}$ discussed in the preceding section, with the appearance of the anti-commutator $\left\{\psi, \psi^{\dagger}\right\}_{+}$a direct result of the effect of the $(-1)^{F}$ factor on the reordering 
of fermion factors inside $\mathbf{T r}$. The time derivative term in Eq. (25b) makes no contribution to the action $\mathbf{S}$, and so the Euler-Lagrange equations following from $\delta \mathbf{S}=0$ can be read off directly from Eq. (25b), giving the dynamical equation for $\psi$

$$
I \hat{D}_{0} \psi=m \psi
$$

together with the constraint

$$
\left\{\psi, \psi^{\dagger}\right\}_{+}=-\lambda_{0}
$$

Equation (25d) is just the standard canonical anti-commutator for a fermion degree of freedom when we identify $-\lambda_{0}=\hbar$ (=1 in microscopic units). We note that this determination of $\lambda_{0}$ has the opposite sign from that found above in the bosonic example; we shall say more about this shortly. Finally, we remark that because the Hilbert space for a single fermion degree of freedom consists of a zero fermion state $|0\rangle \in V_{\mathbf{H}}^{+}$ and a one fermion state $|1\rangle \in V_{\mathbf{H}}^{-}$, the effect of the $(-1)^{F}$ in the definition of $\operatorname{Tr}$ is to give

$$
\operatorname{Tr} 1=1-1=0,
$$

and so taking $\operatorname{Tr}$ of the left- and right-hand sides of Eq. (25d) gives a consistent equation for non-zero $\lambda_{0}$,

$$
0=\operatorname{Tr}\left(\psi \psi^{\dagger}-\psi \psi^{\dagger}\right)=\operatorname{Tr}\left\{\psi, \psi^{\dagger}\right\}_{+}=-\lambda_{0} \operatorname{Tr} 1=-\lambda_{0} 0
$$

From the time derivative term in Eq. (25b), we identify the momentum canonical to $\psi$ as

$$
p_{\psi}=\frac{\delta \mathbf{L}}{\delta \dot{\psi}}=I \psi^{\dagger}
$$

Since by property (ii) of $\operatorname{Tr}$ we have

$$
\operatorname{Tr}\left(I \psi^{\dagger} \dot{\psi}\right)=\operatorname{Tr}\left[\left(\frac{I}{2} \psi^{\dagger} \dot{\psi}\right)+\left(\frac{I}{2} \psi^{\dagger} \dot{\psi}\right)^{\dagger}\right]=\operatorname{Tr}\left[\frac{I}{2}\left(\psi^{\dagger} \dot{\psi}-\dot{\psi}^{\dagger} \psi\right)\right]
$$

the total trace Hamiltonian becomes

$$
\begin{aligned}
\mathbf{H} & =\operatorname{Tr}\left(I \psi^{\dagger} \dot{\psi}\right)-\mathbf{L}=\operatorname{Tr}\left[\frac{I}{2}\left(\psi^{\dagger} \dot{\psi}-\dot{\psi}^{\dagger} \psi\right)\right]-\mathbf{L} \\
& =\operatorname{Tr}\left(I\left[\left\{\psi, \psi^{\dagger}\right\}_{+}+\lambda_{0}\right] B_{0}+m \psi^{\dagger} \psi\right)
\end{aligned}
$$


which simplifies in the Hamiltonian gauge $B_{0}=0$ to

$$
\mathbf{H}=\operatorname{Tr}\left(m \psi^{\dagger} \psi\right)=\operatorname{Tr}\left(-\operatorname{Im} p_{\psi} \psi\right)
$$

Hence taking operator variations, we get

$$
\delta \mathbf{H}=\operatorname{Tr}\left(-\operatorname{Im} \delta p_{\psi} \psi-\operatorname{Im} p_{\psi} \delta \psi\right)=\operatorname{Tr}\left[\operatorname{Im}\left(\psi \delta p_{\psi}-p_{\psi} \delta \psi\right)\right]
$$

and so Eq. (5f) becomes

$$
I \dot{\psi}^{\dagger}=\dot{p}_{\psi}=-\frac{\delta \mathbf{H}}{\delta \psi}=\operatorname{Im} p_{\psi}=-m \psi^{\dagger}, \quad \dot{\psi}=-\frac{\delta \mathbf{H}}{\delta p_{\psi}}=-\operatorname{Im} \psi
$$

in agreement with Eq. (25c) and its adjoint when $B_{0}=0$. As in our bosonic example, in the fermionic case there is also an operator Hamiltonian

$$
H=m \psi^{\dagger} \psi
$$

which, with $\left\{\psi, \psi^{\dagger}\right\}_{+}=-\lambda_{0}$, obeys

$$
\frac{1}{-I \lambda_{0}}[\psi, H]=-\operatorname{Im} \psi=\dot{\psi}, \quad \frac{1}{-I \lambda_{0}}\left[\psi^{\dagger}, H\right]=\operatorname{Im} \psi^{\dagger}=\dot{\psi}^{\dagger}
$$

Hence the dynamics of Eq. (26f) is unitary, again permitting an operator gauge transformation from the Heisenberg picture to the Schrödinger picture.

We see, then, that the standard quantum mechanics of a single bosonic and a single fermionic degree of freedom, including the canonical commutator and anti-commutator, follows from the operator gauge invariant total trace Lagrangian formalism. Let us now examine what happens when more than one degree of freedom is present. Focusing only on the structure of the kinetic terms, for a set $\left\{q_{r}\right\},\left\{\psi_{s}\right\}$ of bosonic and fermionic variables, we have the conventional Lagrangian

$$
L_{\left\{q_{r}\right\},\left\{\psi_{s}\right\}}^{c}=\sum_{r=1}^{R} \frac{1}{2} \dot{q}_{r}^{2}+\sum_{s=1}^{S} \frac{I}{2}\left(\psi_{s}^{\dagger} \dot{\psi}_{s}-\dot{\psi}_{s}^{\dagger} \psi_{s}\right) .
$$

There is now more than one way to extend Eq. (27a) into an operator gauge invariant Lagrangian, depending on whether we require invariance when all variables are subject to the same operator unitary transformation, 
or are subject to independent operator unitary transformations. When all variables are subject to the same transformation,

$$
q_{r} \rightarrow U q_{r} U^{\dagger}, \quad \psi_{s} \rightarrow U \psi_{s} U^{\dagger}, \quad U U^{\dagger}=U^{\dagger} U=1
$$

we achieve covariance of the Lagrangian by replacing $L_{\left\{q_{r}\right\},\left\{\psi_{s}\right\}}^{c}$ by $L_{\left\{q_{r}\right\},\left\{\psi_{s}\right\}}^{(1)}$, with

$$
L_{\left\{q_{r}\right\},\left\{\psi_{s}\right\}}^{(1)}=\sum_{r=1}^{R} \frac{1}{2}\left(\hat{D}_{0} q_{r}\right)^{2}+\sum_{s=1}^{S} \frac{I}{2}\left[\psi_{s}^{\dagger} \hat{D}_{0} \psi_{s}-\left(\hat{D}_{0} \psi_{s}^{\dagger}\right) \psi_{s}\right],
$$

where $\hat{D}_{0}$ acts on the bosonic and fermionic degrees of freedom as in Eqs. (16c) and (24c). The total trace Lagrangian and action

$$
\mathbf{L}_{\left\{q_{r}\right\},\left\{\psi_{s}\right\}}^{(1)}=\operatorname{Tr} L_{\left\{q_{r}\right\},\left\{\psi_{s}\right\}}^{(1)}, \quad \mathbf{S}_{\left\{q_{r}\right\},\left\{\psi_{s}\right\}}^{(1)}=\int_{-\infty}^{\infty} d t \mathbf{L}_{\left\{q_{r}\right\},\left\{\psi_{s}\right\}}^{(1)},
$$

are then invariant under Eq. (27b), and the constraint equation arising from the variation with respect to $B_{0}$ of

$$
\mathbf{S}=\mathbf{S}_{\left\{q_{r}\right\},\left\{\psi_{s}\right\}}^{(1)}-\lambda_{0} \mathbf{S}_{B_{0}}
$$

with $\mathbf{S}_{B_{0}}$ defined as in Eqs. (18b) and (19a), is [cf Eqs. (20b) and (25b)]

$$
\sum_{r=1}^{R}\left[q_{r}, \hat{D}_{0} q_{r}\right]-I \sum_{s=1}^{S}\left\{\psi_{s}, \psi_{s}^{\dagger}\right\}_{+}-\lambda_{0} I=0 .
$$

We see that the individual canonical commutators and anti-commutators are not determined, but only the linear combination given by the sum of bosonic commutators minus the sum of fermionic anti-commutators. The constraint of Eq. (27f), as well as the dynamical equations of motion for $\left\{q_{r}\right\}$ and $\left\{\psi_{s}\right\}$, are consistent with the imposition of the canonical relations

$$
\left[q_{r}, \hat{D}_{0} q_{r}\right]=I, \quad\left\{\psi_{s}, \psi_{s}^{\dagger}\right\}_{+}=1
$$

for each $r$ and $s$, but do not require these. In the language of the conventional theory of constrained systems, Eqs. (27g) are invariant relations which are compatible with the Lagrangian constraints and equations of motion. We note the interesting fact that when the numbers of bosonic and fermionic degrees of freedom are equal, as is the case for a supersymmetric theory, and the canonical relations of Eq. (27g) are imposed, then the constraint of Eq. (27f) is satisfied with $\lambda_{0}=0$. 
An alternative possibility is to require invariance of the total trace Lagrangian under independent operator transformations of the canonical variables,

$$
\begin{gathered}
q_{r} \rightarrow U_{r} q_{r} U_{r}^{\dagger}, \quad \psi_{s} \rightarrow U_{s} \psi_{s} U_{s}^{\dagger}, \\
U_{r} U_{r}^{\dagger}=U_{r}^{\dagger} U_{r}=U_{s} U_{s}^{\dagger}=U_{s}^{\dagger} U_{s}=1 .
\end{gathered}
$$

To achieve this, we introduce an independent covariant derivative for each canonical variable,

$$
\dot{q}_{r} \rightarrow \hat{D}_{0 r} q_{r} \equiv \frac{\partial}{\partial t} q_{r}+\left[B_{0 r}, q_{r}\right], \quad \dot{\psi}_{s} \rightarrow \hat{D}_{0 s} \psi_{s} \equiv \frac{\partial}{\partial t} \psi_{s}+\left[B_{0 s}, \psi_{s}\right]
$$

with $B_{0 r}, r=1, \ldots, R$ and $B_{0 s}, s=1, \ldots, S$ independent anti-self-adjoint operator gauge potentials. We now replace the Lagrangian $L_{\left\{q_{r}\right\},\left\{\psi_{s}\right\}}^{c}$ by

$$
L_{\left\{q_{r}\right\},\left\{\psi_{s}\right\}}^{(2)}=\sum_{r=1}^{R} \frac{1}{2}\left(\hat{D}_{0 r} q_{r}\right)^{2}+\sum_{s=1}^{S} \frac{I}{2}\left[\psi_{s}^{\dagger} \hat{D}_{0 s} \psi_{s}-\left(\hat{D}_{0 s} \psi_{s}^{\dagger}\right) \psi_{s}\right],
$$

the individual terms of which transform covariantly (but now independently) under Eq. (28a),

$$
L_{\left\{q_{r}\right\},\left\{\psi_{s}\right\}}^{(2)} \rightarrow \sum_{r=1}^{R} U_{r} \frac{1}{2}\left(\hat{D}_{0 r} q_{r}\right)^{2} U_{r}^{\dagger}+\sum_{s=1}^{S} U_{s} \frac{I}{2}\left[\psi_{s}^{\dagger} \hat{D}_{0 s} \psi_{s}-\left(\hat{D}_{0 s} \psi_{s}^{\dagger}\right) \psi_{s}\right] U_{s}^{\dagger}
$$

Equation (28d) has the general form of Eq. (8d), and correspondingly, the total trace Lagrangian and action defined by

$$
\mathbf{L}_{\left\{q_{r}\right\},\left\{\psi_{s}\right\}}^{(2)}=\operatorname{Tr} L_{\left\{q_{r}\right\},\left\{\psi_{s}\right\}}^{(2)}, \quad S_{\left\{q_{r}\right\},\left\{\psi_{s}\right\}}^{(2)}=\int_{-\infty}^{\infty} d t \mathbf{L}_{\left\{q_{r}\right\},\left\{\psi_{s}\right\}}^{(2)}
$$

are invariant under Eq. (28a). For each gauge potential $B_{0 r, s}$, we can add an action term $\mathbf{S}_{B_{0 r, s}}$ formed as in Eqs. (18b) and (19a), giving for the overall total trace action

$$
\mathbf{S}=\mathbf{S}_{\left\{q_{r}\right\},\left\{\psi_{s}\right\}}^{(2)}-\sum_{r=1}^{R} \lambda_{0 r} \mathbf{S}_{B_{0 r}}-\sum_{s=1}^{S} \lambda_{0 s} \mathbf{S}_{B_{0 s}}
$$

Varying with respect to each $B_{0 r, s}$, we get the independent constraints

$$
\begin{array}{rr}
{\left[q_{r}, \hat{D}_{0 r} q_{r}\right]-\lambda_{0 r} I=0,} & r=1, \ldots, R, \\
\left\{\psi_{s}, \psi_{s}^{\dagger}\right\}+\lambda_{0 s}=0, & s=1, \ldots, S,
\end{array}
$$


which are closer in structure to the usual canonical commutators than the single constraint of Eq. (27f). Of course, when we impose the requirement of invariance under independent operator transformations as in Eq. (28a), the interaction terms in the total trace Lagrangian are much more tightly restricted in form than when we impose only the global invariance of Eq. (27b). In field theory applications, the indices $r, s$ are typically composite indices, indicating both the spatial coordinate value $\vec{x}$ and the particular field component

at $\vec{x}$. In this case, the constraints associated with $B_{0}$ have a structure intermediate in form between those of Eqs. (27f) and (28g): the constraints at different values of $\vec{x}$ are independent, but at each $\vec{x}$ consist of a sum of contributions from the various bosonic and fermionic field components present in the theory, evaluated at that value of $\vec{x}$.

Various further examples of operator gauge invariant quantum mechanical and quantum field systems in complex quantum mechanics, including models with independent left and right gaugings as in Eq. (9b), and operator gauge invariant extensions of Yang-Mills theory, are given in [1]. In fact, we show there that all of the basic field theory building blocks of the standard model can be imbedded in operator gauge invariant theories; this raises the question of studying operator gauge invariant extensions of the full standard model, to see if useful insights (such as restrictions on the parameters, or new calculational methods) can be obtained.

\section{Operator gauge invariant quaternionic field theories}

We turn now to the construction of operator gauge invariant theories in quaternionic quantum mechanics, which is distinguished from the complex case discussed in the preceding section by the non-existence of a left-acting $I$ which commutes with all operators. For what follows only a few facts about quaternionic Hilbert space are needed, which we now proceed to state. Quaternionic Hilbert space $V_{\mathbf{H}}$ is closed under taking linear combinations of ket vectors when right multiplied by quaternion scalars. A quaternion scalar is a number $q$ of the form

$$
\begin{aligned}
& q=q_{0}+i q_{1}+j q_{2}+k q_{3}, \quad q_{0,1,2,3} \text { real }, \\
& i^{2}=j^{2}=k^{2}=-1, \quad i j=-j i=k, \quad j k=-k j=i, \quad k i=-i k=j,
\end{aligned}
$$

from which one finds that quaternion multiplication, while non-commutative [i.e., $q_{1} q_{2} \neq q_{2} q_{1}$ in general], is 
associative [i.e., $\left.q_{1}\left(q_{2} q_{3}\right)=\left(q_{1} q_{2}\right) q_{3}\right]$. The conjugate $\bar{q}$ and modulus $|q|$ of a quaternion are defined by

$$
\begin{aligned}
\bar{q} & =q_{0}-i q_{1}-j q_{2}-k q_{3}, \\
|q| & =(\bar{q} q)^{1 / 2}=(q \bar{q})^{1 / 2}=\left(q_{0}^{2}+q_{1}^{2}+q_{2}^{2}+q_{3}^{2}\right)^{1 / 2},
\end{aligned}
$$

and by Eq. (29a), the conjugate of a product is the product of the conjugates in reverse order,

$$
\overline{q_{1} q_{2}}=\bar{q}_{2} \bar{q}_{1}
$$

Defining the adjoint in $V_{\mathbf{H}}$ as the quaternion conjugate of the matrix (or operator) transpose, the adjoint of a ket vector is a bra vector, the adjoint of a scalar is its conjugate, and from Eq. (29c) one finds the usual rule that the adjoint of any product of multiple factors is the product of the adjoints of the factors in reverse order. The inner product $\langle f \mid g\rangle$ is quaternion-valued, and so any two inner products $\langle f \mid g\rangle$ and $\left\langle f^{\prime} \mid g^{\prime}\right\rangle$ do not in general commute with one another in quaternionic Hilbert space (whereas in complex quantum mechanics they do). In other words, in quaternionic Hilbert space not only do operators not commute with one another, but their matrix elements as well are non-commutative.

For any complete orthonormal set of states $\{|n\rangle\}$, one defines $[12,13]$ the anti-self-adjoint left-acting algebra $I, J, K$ by

$$
(I, J, K)=\sum_{n}|n\rangle(i, j, k)\langle n|=-\left(I^{\dagger}, J^{\dagger}, K^{\dagger}\right),
$$

which obeys an isomorphic image of the algebra of right-acting quaternion scalars,

$$
\begin{aligned}
& I^{2}=J^{2}=K^{2}=-1=-\sum_{n}|n\rangle\langle n|, \\
& I J=-J I=K, \quad J K=-K J=I, \quad K I=-I K=J .
\end{aligned}
$$

For an arbitrary quaternion operator $\mathcal{O}$ which acts on kets from the left (all quaternion quantum fields discussed below fit this description), we can introduce $[12,13]$ a set of "formally real" components $\mathcal{O}_{A}, A=$ $0,1,2,3$ defined by

$$
\begin{aligned}
& \mathcal{O}_{0}=\frac{1}{4}(\mathcal{O}-I \mathcal{O} I-J \mathcal{O} J-K \mathcal{O} K) \\
& \mathcal{O}_{1}=-\frac{1}{4}(I \mathcal{O}+\mathcal{O} I-J \mathcal{O} K+K \mathcal{O} J),
\end{aligned}
$$




$$
\begin{aligned}
& \mathcal{O}_{2}=-\frac{1}{4}(J \mathcal{O}+\mathcal{O} J-K \mathcal{O} I+I \mathcal{O} K) \\
& \mathcal{O}_{3}=-\frac{1}{4}(K \mathcal{O}+\mathcal{O} K-I \mathcal{O} J+J \mathcal{O} I)
\end{aligned}
$$

so-called because they obey

$$
\mathcal{O}=\mathcal{O}_{0}+I \mathcal{O}_{1}+J \mathcal{O}_{2}+K \mathcal{O}_{3}, \quad\left[\mathcal{O}_{A},(I, J, K)\right]=0, \quad A=0,1,2,3
$$

but they do not in general commute with one another. Finally, we note that $-\frac{1}{2} I,-\frac{1}{2} J,-\frac{1}{2} K$ are a set of anti-self-adjoint generators of a one-dimensional quaternionic irreducible representation [6,14] of the group $S U(2)$; since the smallest non-trivial complex irreducible representation of $S U(2)$ is the two-dimensional spinor representation, this means that any quaternionic quantum field theory based on gauging the onedimensional quaternionic representation of $S U(2)$ is irreducibly quaternionic, and cannot be reduced to a complex quantum field theory. All of the field equations discussed in this section have this character.

With these facts in mind, we are ready to begin the construction of operator gauge invariant quaternionic field models. We begin with the field theory of a quaternionic scalar field $\phi$, which is not restricted to be self-adjoint (or anti-self-adjoint), and which is subjected to independent left and right local gaugings, ${ }^{*}$

$$
\phi \rightarrow U \phi U^{\prime \dagger}, \quad U U^{\dagger}=U^{\dagger} U=U^{\prime} U^{\prime \dagger}=U^{\prime \dagger} U^{\prime}=1 .
$$

Introducing anti-self-adjoint gauge potentials $B_{\mu}, B_{\mu}^{\prime}$ which transform as

$$
B_{\mu} \rightarrow U B_{\mu} U^{\dagger}-\left(\partial_{\mu} U\right) U^{\dagger}, \quad B_{\mu}^{\prime} \rightarrow U^{\prime} B_{\mu}^{\prime} U^{\prime \dagger}-\left(\partial_{\mu} U^{\prime}\right) U^{\prime \dagger}
$$

and the covariant derivative and field strengths

$$
\begin{aligned}
& D_{\mu} \phi=\partial_{\mu} \phi+B_{\mu} \phi-\phi B_{\mu}^{\prime}, \\
& F_{\mu \nu}=\partial_{\mu} B_{\nu}-\partial_{\nu} B_{\mu}+\left[B_{\mu}, B_{\nu}\right], \\
& F_{\mu \nu}^{\prime}=\partial_{\mu} B_{\nu}^{\prime}-\partial_{\nu} B_{\mu}^{\prime}+\left[B_{\mu}^{\prime}, B_{\nu}^{\prime}\right],
\end{aligned}
$$

* An historical discussion of various gaugings which have been proposed for quaternionic fields, and references, is given in Sec. 11.2 of [1].

Our metric convention is $g_{00}=-1, g_{11}=g_{22}=g_{33}=1$. 
which transform as

$$
D_{\mu} \phi \rightarrow U\left(D_{\mu} \phi\right) U^{\prime \dagger}, \quad F_{\mu \nu} \rightarrow U F_{\mu \nu} U^{\dagger}, \quad F_{\mu \nu}^{\prime} \rightarrow U^{\prime} F_{\mu \nu}^{\prime} U^{\prime \dagger}
$$

the operator gauge invariant total trace Lagrangian density for the scalar field is

$$
\begin{aligned}
\mathcal{L} & =\mathcal{L}_{\phi}+\mathcal{L}_{B}+\mathcal{L}_{B^{\prime}}, \\
\mathcal{L}_{\phi} & =\operatorname{Tr}\left\{\frac{1}{2}\left[-\left(D_{\mu} \phi\right)^{\dagger} D^{\mu} \phi-m^{2} \phi^{\dagger} \phi\right]-\frac{\lambda}{4}\left(\phi^{\dagger} \phi\right)^{2}\right\} \\
\mathcal{L}_{B} & =\operatorname{Tr}\left(\frac{1}{4 G^{2}} F_{\nu \mu} F^{\nu \mu}\right), \quad \mathcal{L}_{B^{\prime}}=\operatorname{Tr}\left(\frac{1}{4\left(G^{\prime}\right)^{2}} F_{\nu \mu}^{\prime} F^{\prime \nu \mu}\right) .
\end{aligned}
$$

The total trace Lagrangian $\mathbf{L}$ and action $\mathbf{S}$ are formed from $\mathcal{L}$ by the usual recipe

$$
\mathbf{L}=\int d^{3} x \mathcal{L}, \quad \mathbf{S}=\int d t \mathbf{L} .
$$

Note that an action term analogous to $\mathbf{S}_{B_{0}}$ of Eq. (19a) is now not admissible, because the left-acting $I$ needed to construct this term breaks the operator gauge invariance. When we vary $\mathbf{S}$, through $\delta F_{\mu \nu}$ and $\delta F_{\mu \nu}^{\prime}$ we encounter the covariant derivatives $\hat{D}_{\mu}$ and $\hat{D}_{\mu}^{\prime}$,

$$
\begin{aligned}
& \hat{D}_{\mu} \mathcal{O}=\partial_{\mu} \mathcal{O}+\left[B_{\mu}, \mathcal{O}\right], \\
& \delta F_{\mu \nu}=\hat{D}_{\mu} \delta B_{\nu}-\hat{D}_{\nu} \delta B_{\mu}, \quad \delta F_{\mu \nu}^{\prime}=\hat{D}_{\mu}^{\prime} \delta B_{\nu}^{\prime}-\hat{D}_{\nu}^{\prime} \delta B_{\mu}^{\prime}
\end{aligned}
$$

and in integrating by parts we use the intertwining identities [1]

$$
\begin{aligned}
\hat{D}_{\mu}\left(\rho \eta^{\dagger}\right) & =\left(D_{\mu} \rho\right) \eta^{\dagger}+\rho\left(D_{\mu} \eta\right)^{\dagger}, \\
\hat{D}_{\mu}^{\prime}\left(\rho^{\dagger} \eta\right) & =\left(D_{\mu} \rho\right)^{\dagger} \eta+\rho^{\dagger} D_{\mu} \eta, \\
\partial_{\mu} \operatorname{Tr}\left(\rho \eta^{\dagger}\right) & =\operatorname{Tr}\left[\left(D_{\mu} \rho\right) \eta^{\dagger}+\rho\left(D_{\mu} \eta\right)^{\dagger}\right], \\
\partial_{\mu} \operatorname{Tr}\left(\rho^{\dagger} \eta\right) & =\operatorname{Tr}\left[\left(D_{\mu} \rho\right)^{\dagger} \eta+\rho^{\dagger} D_{\mu} \eta\right],
\end{aligned}
$$

with $\rho$ and $\eta$ either both bosonic or both fermionic in type. Omitting further computational details, we get the operator equations of motion

$$
\begin{array}{ll}
D_{\mu} D^{\mu} \phi-\left(m^{2}+\lambda \phi \phi^{\dagger}\right) \phi & =0, \\
\hat{D}^{\mu} F_{\nu \mu}=G^{2} \mathcal{J}_{\nu}, & \mathcal{J}_{\nu}=\frac{1}{2}\left[\phi\left(D_{\nu} \phi\right)^{\dagger}-\left(D_{\nu} \phi\right) \phi^{\dagger}\right], \\
\hat{D}^{\prime \mu} F_{\nu \mu}^{\prime}=G^{\prime 2} \mathcal{J}_{\nu}^{\prime}, & \mathcal{J}_{\nu}^{\prime}=\frac{1}{2}\left[\phi^{\dagger} D_{\nu} \phi-\left(D_{\nu} \phi\right)^{\dagger} \phi\right],
\end{array}
$$


in which the $\nu=0$ components of the gauge field equations are constraints rather than dynamical equations. Equations (30e) and (32) can be specialized to less general gaugings of $\phi$. For example, if we take $\phi$ to be gauged under

$$
\phi \rightarrow U \phi U^{\dagger}
$$

which is the most general allowed gauging when $\phi$ is self-adjoint, the appropriate Lagrangian density is

$$
\mathcal{L}=\hat{\mathcal{L}}_{\phi}+\mathcal{L}_{B}, \quad \hat{\mathcal{L}}_{\phi}=\operatorname{Tr}\left\{\frac{1}{2}\left[-\left(\hat{D}_{\mu} \phi\right)^{\dagger} \hat{D}^{\mu} \phi-m^{2} \phi^{\dagger} \phi\right]-\frac{\lambda}{4}\left(\phi^{\dagger} \phi\right)^{2}\right\}
$$

and the corresponding equations of motion are

$$
\begin{gathered}
\hat{D}_{\mu} \hat{D}^{\mu} \phi-\left(m^{2}+\lambda \phi \phi^{\dagger}\right) \phi=0 \\
\hat{D}^{\mu} F_{\nu \mu}=\frac{1}{2} G^{2}\left[\phi\left(\hat{D}_{\nu} \phi\right)^{\dagger}-\left(\hat{D}_{\nu} \phi\right) \phi^{\dagger}+\phi^{\dagger} \hat{D}_{\nu} \phi-\left(\hat{D}_{\nu} \phi\right)^{\dagger} \phi\right] .
\end{gathered}
$$

We turn next to the case of quaternionic fermion fields, working always in the Majorana representation for the Dirac matrices, starting again with the most general gauging, in which there are two fermions $\psi_{(1)}, \psi_{(2)}$ transforming under independent left and right local gauge transformations as

$$
\psi_{(1)} \rightarrow U \psi_{(1)} U^{\prime \dagger}, \quad \psi_{(2)} \rightarrow U \psi_{(2)} U^{\prime \dagger}
$$

The operator gauge invariant total trace Lagrangian density is

$$
\mathcal{L}=\mathcal{L}_{\psi_{(1,2)}}+\mathcal{L}_{B}+\mathcal{L}_{B^{\prime}}
$$

with $\mathcal{L}_{B}$ and $\mathcal{L}_{B^{\prime}}$ as in Eq. (30e), and with $\mathcal{L}_{\psi_{(1,2)}}$ given by

$$
\begin{aligned}
\mathcal{L}_{\psi_{(1,2)}}=\operatorname{Tr}\left\{\frac{1}{2}[\right. & \left.\psi_{(2)}^{\dagger} \gamma^{0} \gamma^{\mu} D_{\mu} \psi_{(1)}+\left(D_{\mu} \psi_{(1)}\right)^{\dagger} \gamma^{0} \gamma^{\mu} \psi_{(2)}-\psi_{(1)}^{\dagger} \gamma^{0} \gamma^{\mu} D_{\mu} \psi_{(2)}-\left(D_{\mu} \psi_{(2)}\right)^{\dagger} \gamma^{0} \gamma^{\mu} \psi_{(1)}\right] \\
& \left.+m\left(\psi_{(2)}^{\dagger} i \gamma^{0} \psi_{(1)}-\psi_{(1)}^{\dagger} i \gamma^{0} \psi_{(2)}\right)\right\}
\end{aligned}
$$

Varying the action $\mathbf{S}$ [still related to $\mathcal{L}$ by Eq. (30f)] and recalling that in the Majorana representation $\gamma^{0} \gamma^{\mu}$ and $i \gamma^{0}$ are, respectively, real symmetric and real skew-symmetric matrices, we get the operator equations of motion

$$
\begin{gathered}
\left(\gamma^{0} \gamma^{\mu} D_{\mu}+m i \gamma^{0}\right) \psi_{(1)}=0, \quad\left(\gamma^{0} \gamma^{\mu} D_{\mu}+m i \gamma^{0}\right) \psi_{(2)}=0, \\
\hat{D}^{\mu} F_{\nu \mu}=G^{2} \mathcal{J}_{\nu}, \quad \mathcal{J}_{\nu}=\psi_{(1)}^{T} \gamma_{\nu}^{T} \gamma^{0 T} \psi_{(2)}^{\dagger T}-\psi_{(2)}^{T} \gamma_{\nu}^{T} \gamma^{0 T} \psi_{(1)}^{\dagger T}, \\
\hat{D}^{\prime \mu} F_{\nu \mu}^{\prime}=-\left(G^{\prime}\right)^{2} \mathcal{J}_{\nu}^{\prime}, \quad \mathcal{J}_{\nu}^{\prime}=\psi_{(1)}^{\dagger} \gamma^{0} \gamma_{\nu} \psi_{(2)}-\psi_{(2)}^{\dagger} \gamma^{0} \gamma_{\nu} \psi_{(1)},
\end{gathered}
$$


with $T$ indicating Dirac index (but not operator) transposition. Again, the $\nu=0$ components of the gauge field equations are constraints. As in the boson case, we can readily specialize the two fermion model to the less general gauging

$$
\psi_{(1)} \rightarrow U \psi_{(1)} U^{\dagger}, \quad \psi_{(2)} \rightarrow U \psi_{(2)} U^{\dagger}
$$

for which the appropriate Lagrangian density is

$$
\begin{aligned}
& \mathcal{L}=\hat{\mathcal{L}}_{\psi_{(1,2)}}+\mathcal{L}_{B} \\
& \hat{\mathcal{L}}_{\psi_{(1,2)}}=\operatorname{Tr}\left\{\frac{1}{2}\left[\psi_{(2)}^{\dagger} \gamma^{0} \gamma^{\mu} \hat{D}_{\mu} \psi_{(1)}+\left(\hat{D}_{\mu} \psi_{(1)}\right)^{\dagger} \gamma^{0} \gamma^{\mu} \psi_{(2)}-\psi_{(1)}^{\dagger} \gamma^{0} \gamma^{\mu} \hat{D}_{\mu} \psi_{(2)}-\left(\hat{D}_{\mu} \psi_{(2)}\right)^{\dagger} \gamma^{0} \gamma^{\mu} \psi_{(1)}\right]\right. \\
&+\left.m\left(\psi_{(2)}^{\dagger} i \gamma^{0} \psi_{(1)}-\psi_{(1)}^{\dagger} i \gamma^{0} \psi_{(2)}\right)\right\},
\end{aligned}
$$

and the corresponding equations of motion are

$$
\begin{gathered}
\left(\gamma^{0} \gamma^{\mu} \hat{D}_{\mu}+m i \gamma^{0}\right) \psi_{(1)}=0, \quad\left(\gamma^{0} \gamma^{\mu} \hat{D}_{\mu}+m i \gamma^{0}\right) \psi_{(2)}=0 \\
\hat{D}^{\mu} F_{\nu \mu}=G^{2}\left[\psi_{(1)}^{T} \gamma_{\nu}^{T} \gamma^{0 T} \psi_{(2)}^{\dagger T}-\psi_{(2)}^{T} \gamma_{\nu}^{T} \gamma^{0 T} \psi_{(1)}^{\dagger T}-\psi_{(1)}^{\dagger} \gamma^{0} \gamma_{\nu} \psi_{(2)}+\psi_{(2)}^{\dagger} \gamma^{0} \gamma_{\nu} \psi_{(1)}\right] .
\end{gathered}
$$

We can also form fermionic models with a single fermion field, in which either the left or the right gauge invariance is restricted to be a complex gauge invariance. With the right gauge invariance restricted, we get

$$
\mathcal{L}_{\psi}=\operatorname{Tr}\left\{\frac{I^{\prime}}{2}\left[\psi^{\dagger} \gamma^{0} \gamma^{\mu} D_{\mu} \psi-\left(D_{\mu} \psi\right)^{\dagger} \gamma^{0} \gamma^{\mu} \psi\right]+I^{\prime} m \psi^{\dagger} i \gamma^{0} \psi\right\}
$$

with $I^{\prime}$ a space-time independent left algebra operator, and with $U^{\prime}$ and $B_{\mu}^{\prime}$ restricted to be $\mathbb{C}\left(1, I^{\prime}\right)$. Similarly, with the left gauge invariance restricted, we have

$$
\mathcal{L}_{\psi}^{\prime}=\operatorname{Tr}\left\{\frac{1}{2}\left[\psi^{\dagger} I \gamma^{0} \gamma^{\mu} D_{\mu} \psi-\left(D_{\mu} \psi\right)^{\dagger} \gamma^{0} \gamma^{\mu} I \psi\right]+m \psi^{\dagger} I i \gamma^{0} \psi\right\}
$$

with $I$ a space-time independent left algebra operator, and with $U$ and $B_{\mu}$ restricted to be $\mathbb{C}(1, I)$. When $B_{\mu}$ is so restricted, an action term $\mathbf{S}_{B_{\mu}}$ analogous to Eq. (19a) can be included in the total action, and similarly for $B_{\mu}^{\prime}$ in the case of Eq. (37a).

Before proceeding to the total trace Hamiltonian form of the dynamics, we discuss a number of issues which can be addressed directly from the total trace Lagrangian and the equations of motion. 
(1) We begin by contrasting the quaternionic gauge field structure with that of a conventional Yang-Mills gauge field. Let $1, E_{A}, A=1,2,3$ be a space-time-independent left algebra basis, and let us use Eqs. $(29 f, g)$ to expand the gauge potential $B_{\mu}$ and the corresponding field-strength $F_{\mu \nu}$ over this basis,

$$
B_{\mu}=B_{0 \mu}+\sum_{A=1}^{3} B_{A \mu} E_{A}, \quad F_{\mu \nu}=F_{0 \mu \nu}+\sum_{A=1}^{3} F_{A \mu \nu} E_{A},
$$

with the expansion coefficients $B_{0 \mu}, B_{A \mu}, F_{0 \mu \nu}, F_{A \mu \nu}$ formally real,

$$
\left[B_{0 \mu}, E_{C}\right]=\left[B_{A \mu}, E_{C}\right]=\left[F_{0 \mu \nu}, E_{C}\right]=\left[F_{A \mu \nu}, E_{C}\right]=0
$$

We recall, however, from the discussion beginning the section, that in general the expansion coefficients $B_{0 \mu}, B_{A \mu}, \ldots$ are still operators which do not commute with one another. Substituting Eqs. (38a) into the formula of Eq. (30c) which relates the gauge field strength $F_{\mu \nu}$ to the gauge potential $B_{\mu}$, we find that the expansion coefficients $F_{A \mu \nu}, A=0, \ldots, 3$ are related to the $B_{A \mu}, A=0, \ldots, 3$ by

$$
\begin{aligned}
& F_{0 \mu \nu}=\partial_{\mu} B_{0 \nu}-\partial_{\nu} B_{0 \mu}+\left[B_{0 \mu}, B_{0 \nu}\right]-\sum_{A=1}^{3}\left[B_{A \mu}, B_{A \nu}\right] \\
& F_{A \mu \nu}=\partial_{\mu} B_{A \nu}-\partial_{\nu} B_{A \mu}+\left[B_{A \mu}, B_{0 \nu}\right]-\left[B_{A \nu}, B_{0 \mu}\right]+\sum_{B, C=1}^{3} \varepsilon_{A B C}\left\{B_{B \mu}, B_{C \nu}\right\}_{+} \cdot
\end{aligned}
$$

If $B_{A \mu}, A=0, \ldots, 3$ all commute with one another, Eq. (38c) would reduce to $U(1)$ and $S U(2)$ conventional gauge field structures,

$$
\begin{aligned}
F_{0 \mu} & =\partial_{\mu} B_{0 \nu}-\partial_{\nu} B_{0 \mu} \\
F_{A \mu \nu} & =\partial_{\mu} B_{A \nu}-\partial_{\nu} B_{A \mu}+2 \sum_{B, C=1}^{3} \varepsilon_{A B C} B_{B \mu} B_{C \nu} .
\end{aligned}
$$

But in the general case with non-commuting formally real components $B_{A \mu}$, Eqs. (38c) are not equivalent to Eqs. (38d). Equations (38c) represent only part of the complete system of equations following from the total trace Lagrangians of Eqs. (30e), (34b,c), etc. It is straightforward to reexpress all of the remaining field equations in terms of formally real components with respect to the left-acting algebra $1, E_{A}$

Because the $B_{A \mu}$ are quaternionic operators, they can themselves be expanded over formally real components with respect to a second left-acting algebra $1, E_{B}^{(1)}$ which commutes with $1, E_{A}$,

$$
B_{A \mu}=B_{0 A \mu}+\sum_{B=1}^{3} B_{B A \mu} E_{B}^{(1)}
$$




$$
\begin{aligned}
{\left[B_{B A \mu}, E_{C}\right] } & =\left[B_{B A \mu}, E_{C}^{(1)}\right]=0, \\
{\left[E_{B}, E_{C}^{(1)}\right] } & =0, \quad A, B, C=0,1,2,3 .
\end{aligned}
$$

This process can be continued to any order, giving in $n$th order a multi-quaternion expansion of the form (with $E_{0}=E_{0}^{(1)}=\ldots=E_{0}^{(n)}=1$ )

$$
B_{\mu}=\sum_{A=0}^{3} \sum_{A_{1}=0}^{3} \ldots \sum_{A_{n}=0}^{3} B_{A_{n} \ldots A_{1} A \mu} E_{A} E_{A_{1}}^{(1)} \ldots E_{A_{n}}^{(n)}
$$

with, for all index values, vanishing commutators

$$
\left[E_{B}, E_{C}^{(r)}\right]=\left[E_{B}^{(r)}, E_{C}^{(s)}\right]=\left[B_{A_{n} \ldots A_{1} A \mu}, E_{B}\right]=\left[B_{A_{n} \ldots A_{1} A \mu}, E_{B}^{(r)}\right]=0
$$

Note that the occurrence of left acting multi-quaternion algebras does not imply that probability amplitudes belong to a non-division algebra. The algebra of right-acting scalars remains just $1, i, j, k$, with the possibility of multiple commuting left-acting algebras arising because these are quaternionic Hilbert space operators, rather than scalars. For example, in as small as a four dimensional quaternionic Hilbert space there are two mutually commuting left algebra bases,

$$
\begin{aligned}
1 & =\left[\begin{array}{llll}
1 & 0 & 0 & 0 \\
0 & 1 & 0 & 0 \\
0 & 0 & 1 & 0 \\
0 & 0 & 0 & 1
\end{array}\right], E_{1}=i 1, E_{2}=j 1, E_{3}=k 1, \\
E_{1}^{(1)} & =\left[\begin{array}{cccc}
0 & 0 & 0 & 1 \\
0 & 0 & -1 & 0 \\
0 & 1 & 0 & 0 \\
-1 & 0 & 0 & 0
\end{array}\right], E_{2}^{(1)}=\left[\begin{array}{cccc}
0 & 0 & 1 & 0 \\
0 & 0 & 0 & 1 \\
-1 & 0 & 0 & 0 \\
0 & -1 & 0 & 0
\end{array}\right], E_{3}^{(1)}=\left[\begin{array}{cccc}
0 & -1 & 0 & 0 \\
1 & 0 & 0 & 0 \\
0 & 0 & 0 & 1 \\
0 & 0 & -1 & 0
\end{array}\right] .
\end{aligned}
$$

Properties of multi-quaternion algebras have been studied in a series of papers by Razon and Horwitz [15-17], and by various authors [18-21] who classified the $U(2)$ case of the "color charge algebras" introduced by Adler [22]. (The color charge algebras correspond to multi-quaternion bases $E_{A_{1}}^{(1)} \ldots E_{A_{n}}^{(n)}$, in which the indices $A_{1}, \ldots, A_{n}$ are contracted so as to leave a single free index $A$.) The latter calculations indicate that large Lie algebras are readily built up from multi-quaternion bases.

(2) In the fermionic Lagrangians of Eqs. (34c) and (36b), the gauge bosons couple to vector currents only, and so there are no chiral anomalies and also no Witten [23] anomalies. Since the $\gamma_{5}$ matrix in Majorana representation is imaginary, attempting to split Eqs. (34c) and (36b) into chiral components 
would break the operator gauge invariance. In other words, insisting on a maximal operator gauge invariance in quaternionic field theory excludes chiral fermions.

At the same time, since $i \gamma_{5}$ is real and anti-self-adjoint, when the mass $m$ is zero the fermion total trace Lagrangians written above are invariant under

$$
\begin{aligned}
\psi_{(1,2)} & \rightarrow e^{i \gamma_{5} \beta} \psi_{(1,2)}, & \psi_{(1,2)}^{\dagger} \rightarrow \psi_{(1,2)}^{\dagger} e^{-i \gamma_{5} \beta}, \\
\psi & \rightarrow e^{i \gamma_{5} \beta} \psi, & \psi^{\dagger} \rightarrow \psi^{\dagger} e^{-i \gamma_{5} \beta},
\end{aligned}
$$

with $\beta$ a real $c$-number which is independent of $x$. Thus, when $m=0$, the fermion models all have a chiral symmetry.

(3) Let us next address the issues of the spin-statistics connection and discrete symmetries in quaternionic field theory. On spin and statistics, we have little to say, beyond the fact that the conventional spinstatistics connection has been assumed in including the $(-1)^{F}$ factor in the definition of $\operatorname{Tr}$. We have not made a study of the possibility of an abnormal spin-statistics connection, but note that this would lead to the usual pathologies upon specializing a quaternionic field theory back to a complex one.

To study discrete symmetries of the Lagrangians written down above, we use some standard Dirac matrix apparatus. We begin with parity $(P)$, and readily find that under the substitutions

$$
\begin{aligned}
B_{0}\left(\vec{x}, x^{0}\right) & \rightarrow B_{0}\left(-\vec{x}, x^{0}\right), \quad B_{0}^{\prime}\left(\vec{x}, x^{0}\right) \rightarrow B_{0}^{\prime}\left(-\vec{x}, x^{0}\right), \\
B_{\ell}\left(\vec{x}, x^{0}\right) & \rightarrow-B_{\ell}\left(-\vec{x}, x^{0}\right), \quad B_{\ell}^{\prime}\left(\vec{x}, x^{0}\right) \rightarrow-B_{\ell}^{\prime}\left(-\vec{x}, x^{0}\right), \\
\phi\left(\vec{x}, x^{0}\right) & \rightarrow \eta_{P} \phi\left(-\vec{x}, x^{0}\right), \\
\psi_{(1,2)}\left(\vec{x}, x^{0}\right) & \rightarrow \eta_{P}^{\prime} i \gamma^{0} \psi_{(1,2)}\left(-\vec{x}, x^{0}\right), \quad \psi\left(\vec{x}, x^{0}\right) \rightarrow \eta_{P}^{\prime \prime} i \gamma^{0} \psi\left(-\vec{x}, x^{0}\right),
\end{aligned}
$$

with $\eta_{P}, \eta_{P}^{\prime}$, and $\eta_{P}^{\prime \prime}$ arbitrary real $c$-number phases, all of the total trace Lagrangian densities of this section transform as

$$
\mathcal{L}\left(\vec{x}, x^{0}\right) \rightarrow \mathcal{L}\left(-\vec{x}, x^{0}\right)
$$

and the corresponding total trace actions are invariant. Turning next to time reversal $(T)$, the relevant substitutions are now

$$
B_{0}\left(\vec{x}, x^{0}\right) \rightarrow-B_{0}\left(\vec{x},-x^{0}\right), \quad B_{0}^{\prime}\left(\vec{x}, x^{0}\right) \rightarrow-B_{0}^{\prime}\left(\vec{x},-x^{0}\right)
$$




$$
\begin{aligned}
B_{\ell}\left(\vec{x}, x^{0}\right) & \rightarrow B_{\ell}\left(\vec{x},-x^{0}\right), \quad B_{\ell}^{\prime}\left(\vec{x}, x^{0}\right) \rightarrow B_{\ell}^{\prime}\left(\vec{x},-x^{0}\right), \\
\phi\left(\vec{x}, x^{0}\right) & \rightarrow \eta_{T} \phi\left(\vec{x},-x^{0}\right), \\
\psi_{(1)}\left(\vec{x}, x^{0}\right) & \rightarrow \eta_{T}^{\prime} A \psi_{(1)}\left(\vec{x},-x^{0}\right), \\
\psi_{(2)}\left(\vec{x}, x^{0}\right) & \rightarrow-\eta_{T}^{\prime} A \psi_{(2)}\left(\vec{x},-x^{0}\right), \\
\psi\left(\vec{x}, x^{0}\right) & \rightarrow\left\{\begin{array}{l}
\eta_{T}^{\prime \prime} A \psi\left(\vec{x},-x^{0}\right) J^{\prime} \text { for } \mathcal{L}_{\psi} \\
\eta_{T}^{\prime \prime} J A \psi\left(\vec{x},-x^{0}\right) \text { for } \mathcal{L}_{\psi}^{\prime},
\end{array},\right.
\end{aligned}
$$

with $\eta_{T}, \eta_{T}^{\prime}, \eta_{T}^{\prime \prime}$ arbitrary real $c$-number phases, with $A$ the Majorana representation time reversal matrix, which is real, and with $J^{\prime}, J$ anti-commuting respectively with $I^{\prime}, I$. Under these substitutions all of the total trace Lagrangian densities of this section transform as

$$
\mathcal{L}\left(\vec{x}, x^{0}\right) \rightarrow \mathcal{L}\left(\vec{x},-x^{0}\right)
$$

and the corresponding total trace actions are again invariant.

Turning finally to charge conjugation $(C)$, we consider the substitutions

$$
\begin{aligned}
B_{\mu} & \rightarrow B_{\mu}^{\prime}, \quad B_{\mu}^{\prime} \rightarrow B_{\mu}, \\
\phi & \rightarrow \eta_{C} \phi^{\dagger}, \\
\psi_{(1)} & \rightarrow \eta_{C}^{\prime} \psi_{(1)}^{\dagger T}, \quad \psi_{(2)} \rightarrow-\eta_{C}^{\prime} \psi_{(2)}^{\dagger T}, \\
\psi & \rightarrow \eta_{C}^{\prime \prime} \psi^{\dagger T},
\end{aligned}
$$

under which the covariant derivatives $D_{\mu} \phi, D_{\mu} \psi_{(1,2)}$, and $D_{\mu} \psi$ transform as

$$
D_{\mu} \phi \rightarrow \eta_{C}\left(D_{\mu} \phi\right)^{\dagger}, \quad D_{\mu} \psi_{(1,2)} \rightarrow(+,-) \eta_{C}^{\prime}\left(D_{\mu} \psi_{(1,2)}\right)^{\dagger T}, \quad D_{\mu} \psi \rightarrow \eta_{C}^{\prime \prime}\left(D_{\mu} \psi\right)^{\dagger T}
$$

with $\eta_{C}, \eta_{C}^{\prime}$, and $\eta_{C}^{\prime \prime}$ arbitrary real $c$-number phases and with $T$ the Dirac index transpose. When we impose a condition of equality on the gauge field couplings,

$$
G=G^{\prime}
$$

all of the total trace Lagrangian densities of this section are invariant under the substitutions of Eq. (42a),

$$
\mathcal{L} \rightarrow \mathcal{L}
$$


Since the gauge potentials $B_{\mu}$ and $B_{\mu}^{\prime}$ are interchanged by the substitutions of Eq. (42a), they are not $C$ eigenstates. We see, then, that when the requirement of $C$ invariance is imposed, the models with independent left and right gaugings are left with a single coupling constant $G$. $^{*}$

(4) By using the fact that $i \gamma_{5}$ is real and anti-self-adjoint, we can construct a model with self-adjoint Lagrangian using a single fermion field, without breaking the bi-unitary operator gauge invariance. The Lagrangian density for this model is

$$
\mathcal{L}_{\psi}^{5}=\operatorname{Tr}\left\{\frac{1}{2} \psi^{\dagger} \gamma^{0} \gamma^{\mu}\left(i \gamma_{5}\right) D_{\mu} \psi-\left(D_{\mu} \psi\right)^{\dagger} \gamma^{0} \gamma^{\mu}\left(i \gamma_{5}\right) \psi\right\}
$$

we do not include a mass term because, since $i \gamma_{5}$ anti-commutes with $i \gamma^{0}$, the expression

$$
\psi^{\dagger} i \gamma^{0} i \gamma_{5} \psi
$$

is anti-self-adjoint, and vanishes inside Tr. It is easy to check that under the transformations

$$
\begin{array}{rlrl}
P: & \psi\left(\vec{x}, x^{0}\right) & \rightarrow \eta_{P} i \gamma^{0} \psi\left(-\vec{x}, x^{0}\right), \\
C: & \psi \rightarrow \eta_{C} \psi^{\dagger T}, \\
T: & \psi\left(\vec{x}, x^{0}\right) \rightarrow \eta_{T} A \psi\left(\vec{x},-x^{0}\right),
\end{array}
$$

together with the gluon sector transformations discussed above, the Lagrangian density $\mathcal{L}_{\psi}^{5}$ is $P$ odd, $C$ odd, and $T$ even. Although naively leading to conserved source currents for the gauge gluons, the model $\mathcal{L}_{\psi}^{5}$ has chiral anomalies in the usual complex canonical quantization, which suggests that it may also be inconsistent in the more general total trace Lagrangian dynamics. This question requires further study.

* When $G \neq G^{\prime}$, the models do not have a $C$ or a $C P T$ symmetry; this does not contradict the usual $C P T$ theorem because we do not make the locality assumption that the fields commute at spacelike separations. In [1] it is shown that when the Lagrangians of this section are reinterpreted as complex field theory Lagrangians through a decomposition of the fields into symplectic components, then one finds a $C$ and a $C P T$ symmetry even for $G \neq G^{\prime}$, in agreement with the usual $C P T$ theorem. 
(5) Up to this point, our entire discussion has dealt with field theories in flat space-time. Since the total trace Lagrangians of this section are all Lorentz invariant, they can be generalized to curved spacetime by the standard prescription of replacing the Minkowski metric by a general metric $g_{\mu \nu}$, ordinary derivatives $\partial_{\lambda}$ by covariant derivatives $\nabla_{\lambda}$ which commute with $g_{\mu \nu}$, etc. When this is done, the source term for the gravitational field equation will be a total trace energy-momentum tensor $\mathbf{T}^{\mu \nu}$, defined by computing the variation of the total trace action $\mathbf{S}$ under an infinitesimal metric variation $g_{\mu \nu} \rightarrow g_{\mu \nu}+\delta g_{\mu \nu}$, according to

$$
\mathbf{S} \rightarrow \mathbf{S}+\delta \mathbf{S}, \quad \delta \mathbf{S}=\frac{1}{2} \int d^{4} x[g(x)]^{1 / 2} \mathbf{T}^{\mu \nu}(x) \delta g_{\mu \nu}(x)
$$

(Here $g(x)$ is the negative of the determinant of the metric, and our notation follows Weinberg [24].) Standard arguments then show that $\mathbf{T}^{\mu \nu}$ is covariantly conserved,

$$
\nabla \mu \mathbf{T}^{\mu \nu}=0
$$

and, in the flat space-time limit, the spatial integrals of the various components of $\mathbf{T}^{\mu \nu}$ give the total trace Poincaré generators, for example,

$$
\mathbf{H}=\int d^{3} x \mathbf{T}^{00}
$$

We conclude that quaternionic field theories described by total trace actions can be consistently coupled to classical gravitation, but the total trace structure of the gravitational source term differs from that customarily assumed in the semi-classical theory of gravitation.

(6) It is interesting to ask whether the Lagrangian $\mathbf{L}$ of Eqs. (30f) and (34b,c) has fermionic symmetries, constructed in analogy with the fermionic symmetry of supersymmetric Yang-Mills theory [25,26]. This question can be investigated by studying the change of $\mathbf{L}$ under field variations parameterized by fermionic parameters, leading to a lengthy calculation, the results of which are compactly summarized as covariant divergence equations for the corresponding Noether currents. Let $\mathcal{S}_{(1,2)}^{\lambda}$ be the fermionic currents

$$
\mathcal{S}_{(1,2)}^{\lambda}=\frac{1}{2}\left[\psi_{(1,2)}^{\dagger} \frac{F^{\mu \nu}}{G^{2}}+\frac{F^{\prime \mu \nu}}{G^{\prime 2}} \psi_{(1,2)}^{\dagger}\right] \gamma^{0} \gamma^{\lambda} \frac{1}{2}\left[\gamma_{\mu}, \gamma_{\nu}\right]
$$


which transform under operator gauge transformations as

$$
\mathcal{S}_{(1,2)}^{\lambda} \rightarrow U^{\prime} \mathcal{S}_{(1,2)}^{\lambda} U^{\dagger}
$$

Let us define the covariant derivative $\bar{D}_{\lambda}$ acting on a general operator $\mathcal{O}$ as

$$
\bar{D}_{\lambda} \mathcal{O}=\partial_{\lambda} \mathcal{O}+B_{\lambda}^{\prime} \mathcal{O}-\mathcal{O} B_{\lambda}
$$

so that when $\mathcal{O}$ gauge transforms as

$$
\mathcal{O} \rightarrow U^{\prime} \mathcal{O} U^{\dagger}
$$

$D_{\lambda} \mathcal{O}$ transforms covariantly as

$$
D_{\lambda} \mathcal{O} \rightarrow U^{\prime}\left(D_{\lambda} \mathcal{O}\right) U^{\dagger}
$$

Comparing Eq. (44c) with the definition of $D_{\mu}$ in Eq. (30c), we evidently have

$$
\left(D_{\lambda} \mathcal{O}\right)^{\dagger}=\bar{D}_{\lambda} \mathcal{O}^{\dagger}
$$

Forming the covariant divergence $\bar{D}_{\lambda} \mathcal{S}_{(1,2)}^{\lambda}$ and using Eq. (44f), we get the identity

$$
\begin{gathered}
\bar{D}_{\lambda} \mathcal{S}_{(1,2)}^{\lambda}=\frac{1}{2}\left[\left(D_{\lambda} \psi_{(1,2)}\right)^{\dagger} \frac{F^{\mu \nu}}{G^{2}}+\frac{F^{\prime \mu \nu}}{G^{\prime 2}}\left(D_{\lambda} \psi_{(1,2)}^{\dagger}\right)\right] \gamma^{0} \gamma^{\lambda} \frac{1}{2}\left[\gamma_{\mu}, \gamma_{\nu}\right] \\
+\frac{1}{2}\left[\psi_{(1,2)}^{\dagger} \frac{\left(\hat{D}_{\lambda} F^{\mu \nu}\right)}{G^{2}}+\frac{\left(\hat{D}_{\lambda}^{\prime} F^{\prime \mu \nu}\right)}{G^{\prime 2}} \psi_{(1,2)}^{\dagger}\right] \gamma^{0} \gamma^{\lambda} \frac{1}{2}\left[\gamma_{\mu}, \gamma_{\nu}\right] .
\end{gathered}
$$

The first line of Eq. (45a) can be simplified by using the Dirac equations of Eq. (35), expressed in the form

$$
\left(D_{\lambda} \psi_{(1,2)}\right)^{\dagger} \gamma^{0} \gamma^{\lambda}=\psi_{(1,2)}^{\dagger} m i \gamma^{0}
$$

to give

$$
\frac{1}{2}\left[\psi_{(1,2)}^{\dagger} \frac{F^{\mu \nu}}{G^{2}}+\frac{F^{\prime \mu \nu}}{G^{\prime 2}} \psi_{(1,2)}^{\dagger}\right] \operatorname{mi\gamma }^{0} \frac{1}{2}\left[\gamma_{\mu}, \gamma_{\nu}\right]
$$

The second line of Eq. (45a) can be rearranged by substituting the identity

$$
\gamma^{\lambda} \frac{1}{2}\left[\gamma_{\mu}, \gamma_{\nu}\right]=\gamma_{\mu} \delta_{\nu}^{\lambda}-\gamma_{\nu} \delta_{\mu}^{\lambda}-\varepsilon_{\mu \nu}^{\lambda \alpha} \gamma_{\alpha} i \gamma^{5}
$$


The contribution from $\varepsilon_{\mu \nu}^{\lambda \alpha}$ vanishes by virtue of the Bianchi identities

$$
\hat{D}_{\lambda} F_{\mu \nu}+\hat{D}_{\nu} F_{\lambda \mu}+\hat{D}_{\mu} F_{\nu \lambda}=0, \quad \hat{D}_{\lambda}^{\prime} F_{\mu \nu}^{\prime}+\hat{D}_{\nu}^{\prime} F_{\lambda \mu}^{\prime}+\hat{D}_{\mu}^{\prime} F_{\nu \lambda}^{\prime}=0
$$

The contribution from the Kronecker delta terms is

$$
\left[\psi_{(1,2)}^{\dagger} \frac{\hat{D}_{\nu} F^{\mu \nu}}{G^{2}}+\frac{\hat{D}_{\nu}^{\prime} F^{\prime \mu \nu}}{G^{\prime 2}} \psi_{(1,2)}^{\dagger}\right] \gamma^{0} \gamma_{\mu}
$$

which can be simplified using the gauge field equations of Eq. (35) to give

$$
\left[\psi_{(1,2)}^{\dagger} \mathcal{J}^{\mu}-\mathcal{J}^{\prime \mu} \psi_{(1,2)}^{\dagger}\right] \gamma^{0} \gamma_{\mu}
$$

Thus, putting everything together, we have

$$
\begin{gathered}
\bar{D}_{\lambda} \mathcal{S}_{(1,2)}^{\lambda}=\frac{1}{2}\left(\psi_{(1,2)}^{\dagger} \frac{F^{\mu \nu}}{G^{2}}+\frac{F^{\prime \mu \nu}}{G^{2}} \psi_{(1,2)}^{\dagger}\right) m i \gamma^{0} \frac{1}{2}\left[\gamma_{\mu}, \gamma_{\nu}\right] \\
+\left(\psi_{(1,2)}^{\dagger} \mathcal{J}^{\mu}-\mathcal{J}^{\prime \mu} \psi_{(1,2)}^{\dagger}\right) \gamma^{0} \gamma_{\mu}
\end{gathered}
$$

and we see that even when the fermion mass $m$ vanishes, the fermionic currents $\mathcal{S}_{(1,2)}^{\lambda}$ are not covariantly conserved. Suppose, however, that there is either an operator gauge, or an asymptotic limit, in which the fermion fields $\psi_{(1,2)}$ have the standard canonical anti-commutators of complex fields. In such a situation, we see from Eq. (35) that we would have $\mathcal{J}^{\mu}=\mathcal{J}^{\prime \mu}$, and the second line of Eq. (46a) would reduce to the singular commutator

$$
\left[\psi_{(1,2)}^{\dagger}, \mathcal{J}^{\mu}\right] \gamma^{0} \gamma_{\mu}
$$

which vanishes in dimensional regularization. This argument suggests that Eq. (46a), despite the presence of the gluon source current terms, may nonetheless have useful content.

Let us now return to our main theme of total trace operator dynamics, and construct the total trace Hamiltonian form of the dynamics following from the scalar field Lagrangian of Eqs. (30e,f) and the fermion field Lagrangian of Eqs. (34b,c). From Eq. (30e), we get

$$
\begin{aligned}
p_{\phi} & =\frac{\delta \mathbf{L}}{\delta \dot{\phi}}=\left(D_{0} \phi\right)^{\dagger} \\
p_{B_{\ell}} & =\frac{\delta \mathbf{L}}{\delta \dot{B}_{\ell}}=-\frac{1}{G^{2}} F_{0 \ell}, \quad p_{B_{\ell}^{\prime}}=\frac{\delta \mathbf{L}}{\delta \dot{B}_{\ell}^{\prime}}=-\frac{1}{G^{\prime 2}} F_{0 \ell}^{\prime}
\end{aligned}
$$


and so the total trace Hamiltonian density becomes

$$
\mathcal{H}=\operatorname{Tr}\left[\left(D_{0} \phi\right)^{\dagger} \dot{\phi}-\frac{1}{G^{2}} \sum_{\ell=1}^{3} F_{0 \ell} \dot{B}_{\ell}-\frac{1}{G^{\prime 2}} \sum_{\ell=1}^{3} F_{0 \ell}^{\prime} \dot{B}_{\ell}^{\prime}\right]-\mathcal{L}
$$

Substituting

$$
\begin{aligned}
\dot{\phi} & =D_{0} \phi-B_{0} \phi+\phi B_{0}^{\prime}, \\
\dot{B}_{\ell} & =F_{0 \ell}+\hat{D}_{\ell} B_{0}, \quad \dot{B}_{\ell}^{\prime}=F_{0 \ell}^{\prime}+\hat{D}_{\ell}^{\prime} B_{0}^{\prime},
\end{aligned}
$$

forming the total trace Hamiltonian $\mathbf{H}$ and doing a spatial integration by parts, we get

$$
\mathbf{H}=\int d^{3} x \mathcal{H}=\mathbf{H}_{\phi}+\mathbf{H}_{B, \mathcal{J}_{0}}+\mathbf{H}_{B^{\prime}, \mathcal{J}_{0}^{\prime}}
$$

with

$$
\begin{aligned}
& \mathbf{H}_{\phi}=\int d^{3} x \operatorname{Tr}\left[\frac{1}{2} p_{\phi}^{\dagger} p_{\phi}+\frac{1}{2} \sum_{\ell=1}^{3}\left(D_{\ell} \phi\right)^{\dagger}\left(D_{\ell} \phi\right)+\frac{1}{2} m^{2} \phi^{\dagger} \phi+\frac{\lambda}{4}\left(\phi^{\dagger} \phi\right)^{2}\right], \\
& \mathbf{H}_{B, \mathcal{J}_{0}}=\int d^{3} x \operatorname{Tr}\left[-\frac{G^{2}}{2} \sum_{\ell=1}^{3}\left(p_{B_{\ell}}\right)^{2}-\frac{1}{2 G^{2}} \sum_{\substack{\ell, m=1 \\
\ell<m}}^{3}\left(F_{\ell m}\right)^{2}-B_{0}\left(\mathcal{J}_{0}+\sum_{\ell=1}^{3} \hat{D}_{\ell} p_{B_{\ell}}\right)\right], \\
& \mathbf{H}_{B^{\prime}, \mathcal{J}_{0}^{\prime}}=\int d^{3} x \operatorname{Tr}\left[-\frac{G^{\prime 2}}{2} \sum_{\ell=1}^{3}\left(p_{B_{\ell}^{\prime}}\right)^{2}-\frac{1}{2 G^{\prime 2}} \sum_{\substack{\ell, m=1 \\
\ell<m}}^{3}\left(F_{\ell m}^{\prime}\right)^{2}-B_{0}^{\prime}\left(\mathcal{J}_{0}^{\prime}+\sum_{\ell=1}^{3} \hat{D}_{\ell}^{\prime} p_{B_{\ell}^{\prime}}\right)\right],
\end{aligned}
$$

and with $\mathcal{J}_{0}, \mathcal{J}_{0}^{\prime}$ the 0 components of the boson source currents $\mathcal{J}_{\nu}, \mathcal{J}_{\nu}^{\prime}$ given in Eq. (32). Proceeding similarly in the fermion case, from Eqs. $(34 \mathrm{~b}, \mathrm{c})$ we get

$$
p_{\psi_{(1)}}=\frac{\delta \mathbf{L}}{\delta \dot{\psi}_{(1)}}=\psi_{(2)}^{\dagger}, \quad p_{\psi_{(2)}}=\frac{\delta \mathbf{L}}{\delta \dot{\psi}_{(2)}}=-\psi_{(1)}^{\dagger},
$$

and so the total trace Hamiltonian density becomes

$$
\mathcal{H}=\operatorname{Tr}\left[\psi_{(2)}^{\dagger} \dot{\psi}_{(1)}-\psi_{(1)}^{\dagger} \dot{\psi}_{(2)}-\frac{1}{G^{2}} \sum_{\ell=1}^{3} F_{0 \ell} \dot{B}_{\ell}-\frac{1}{G^{2}} \sum_{\ell=1}^{3} F_{0 \ell}^{\prime} \dot{B}_{\ell}^{\prime}\right]-\mathcal{L}
$$

Substituting

$$
\dot{\psi}_{(1,2)}=D_{0} \psi_{(1,2)}-B_{0} \psi_{(1,2)}+\psi_{(1,2)} B_{0}^{\prime}
$$

together with the second line of Eq. (47c), forming the total trace Hamiltonian and doing a spatial integration by parts, we now get

$$
\mathbf{H}=\mathbf{H}_{\psi_{(1,2)}}+\mathbf{H}_{B, \mathcal{J}_{0}}+\mathbf{H}_{B^{\prime},-\mathcal{J}_{0}^{\prime}},
$$


with

$$
\begin{aligned}
\mathbf{H}_{\psi_{(1,2)}} & =\int d^{3} x \operatorname{Tr}\left\{-\frac{1}{2} \sum_{\ell=1}^{3}\left[\psi_{(2)}^{\dagger} \gamma^{0} \gamma^{\ell} D_{\ell} \psi_{(1)}+\left(D_{\ell} \psi_{(1)}\right)^{\dagger} \gamma^{0} \gamma^{\ell} \psi_{(2)}\right.\right. \\
-\psi_{(1)}^{\dagger} & \left.\left.\gamma^{0} \gamma^{\ell} D_{\ell} \psi_{(2)}-\left(D_{\ell} \psi_{(2)}\right)^{\dagger} \gamma^{0} \gamma^{\ell} \psi_{(1)}\right]-m\left[\psi_{(2)}^{\dagger} i \gamma^{0} \psi_{(1)}-\psi_{(1)}^{\dagger} i \gamma^{0} \psi_{(2)}\right]\right\} .
\end{aligned}
$$

In Eq. (50a), $\mathbf{H}_{B, \mathcal{J}_{0}}$ and $\mathbf{H}_{B^{\prime},-\mathcal{J}_{0}^{\prime}}$ are still given by Eq. (48b) with the substitution $\mathcal{J}_{0}^{\prime} \rightarrow-\mathcal{J}_{0}^{\prime}$, but now $\mathcal{J}_{0}$, $\mathcal{J}_{0}^{\prime}$ are the 0 components of the fermion source currents $\mathcal{J}_{\nu}, \mathcal{J}_{\nu}^{\prime}$ given in Eq. (35).

The total trace Hamiltonian dynamics for $\phi, \psi_{(1)}$, and $\psi_{(2)}$ now takes the form of Eq. (5f), with no further complications. For the gauge potentials $B_{\mu}$ and $B_{\mu}^{\prime}$, however, we encounter the familiar problem that we are dealing with a constrained system, and so the canonical momenta are not independent. Focusing henceforth on the potential $B_{\mu}$ (the treatment of $B_{\mu}^{\prime}$ is completely analogous), we have a primary constraint

$$
p_{B_{0}}=-\frac{1}{G^{2}} F_{00}=0
$$

which is satisfied as an identity without use of the equations of motion. Differentiating Eq. (51a) with respect to time, we get the secondary constraint

$$
0=\dot{p}_{B_{0}}=-\frac{\delta}{\delta B_{0}} \mathbf{H}_{B, \mathcal{J}_{0}}=\mathcal{J}_{0}+\sum_{\ell=1}^{3} \hat{D}_{\ell} p_{B_{\ell}},
$$

which is the same as the constraint arising from the Lagrangian equations of motion. Further time differentiation of Eq. (51b) leads to no further secondary constraints, since the equation

$$
\dot{\mathcal{J}}_{0}+\frac{\partial}{\partial t}\left(\sum_{\ell=1}^{3} \hat{D}_{\ell} p_{B_{\ell}}\right)=0
$$

can be rearranged [with use of Eq. (51b) and properties of the covariant derivative $\hat{D}_{\mu}$ ] into the form

$$
\hat{D}^{\mu} \mathcal{J}_{\mu}=0
$$

which is satisfied by virtue of the Lagrangian equations of motion for $\phi$ or $\psi_{(1,2)}$. The constraint structure is thus completely analogous to that of a conventional Yang-Mills gauge field, for which the simplest way to realize a Hamiltonian dynamics is to use axial gauge $[27,28]$, in which $B_{3}$ is taken to vanish,

$$
B_{3}=0
$$


In this gauge we have

$$
\begin{gathered}
F_{03}=\partial_{0} B_{3}-\partial_{3} B_{0}+\left[B_{0}, B_{3}\right]=-\partial_{3} B_{0}, \\
\hat{D}_{3} F_{03}=\partial_{3} F_{03}+\left[B_{3}, F_{03}\right]=-\partial_{3}^{2} B_{0}, \\
F_{13}=-\partial_{3} B_{1}, \quad F_{23}=-\partial_{3} B_{2},
\end{gathered}
$$

and so the constraint

$$
G^{2} \mathcal{J}_{0}=\sum_{\ell=1}^{3} \hat{D}_{\ell}\left(-G^{2} p_{B_{\ell}}\right)=\sum_{\ell=1}^{3} \hat{D}_{\ell} F_{0 \ell}=\hat{D}_{1} F_{01}+\hat{D}_{2} F_{02}-\partial_{3}^{2} B_{0}
$$

can be directly integrated to yield $B_{0}$ and $F_{03}$, giving (with $x_{3}=z$ )

$$
\begin{gathered}
B_{0}=-\frac{1}{2} G^{2} \int_{-\infty}^{\infty} d z^{\prime}\left|z-z^{\prime}\right|\left(\mathcal{J}_{0}+\hat{D}_{1} p_{B_{1}}+\hat{D}_{2} p_{B_{2}}\right)_{z^{\prime}} \\
F_{03}=\frac{1}{2} G^{2} \int_{-\infty}^{\infty} d z^{\prime} \frac{z-z \prime}{\left|z-z^{\prime}\right|}\left(\mathcal{J}_{0}+\hat{D}_{1} p_{B_{1}}+\hat{D}_{2} p_{B_{2}}\right)_{z^{\prime}} .
\end{gathered}
$$

Substituting Eqs. (51b) and (52b) back into $\mathbf{H}_{B, \mathcal{J}_{0}}$, we get

$$
\begin{aligned}
\mathbf{H}_{B, \mathcal{J}_{0}}=\int d^{3} x & \operatorname{Tr}\left\{-\frac{G^{2}}{2} \sum_{\ell=1,2}\left(p_{B_{\ell}}\right)^{2}-\frac{1}{2 G^{2}}\left(F_{03}\right)^{2}-\frac{1}{2 G^{2}}\left(\partial_{1} B_{2}-\partial_{2} B_{1}+\left[B_{1}, B_{2}\right]\right)^{2}\right. \\
- & \left.\frac{1}{2 G^{2}}\left(\partial_{3} B_{1}\right)^{2}-\frac{1}{2 G^{2}}\left(\partial_{3} B_{2}\right)^{2}\right\}
\end{aligned}
$$

with $F_{03}$ given by Eq. (52d), and so only $B_{1}, B_{2}$ remain as independent dynamical degrees of freedom, with the corresponding independent canonical momenta $p_{B_{1}}, p_{B_{2}}$. It is now completely straightforward to verify that the operator equations of motion obtained from the total trace Hamiltonian $\mathbf{H}_{B, \mathcal{J}_{0}}$ of Eq. (52e),

$$
\begin{array}{rlrl}
\frac{\delta \mathbf{H}_{B, \mathcal{J}_{0}}}{\delta p_{B_{1}}}=\partial_{0} B_{1}, & & \frac{\delta \mathbf{H}_{B, \mathcal{J}_{0}}}{\delta p_{B_{2}}}=\partial_{0} B_{2}, \\
\frac{\delta \mathbf{H}_{B, \mathcal{J}_{0}}}{\delta B_{1}}=-\dot{p}_{B_{1}}, & \frac{\delta \mathbf{H}_{B, \mathcal{J}_{0}}}{\delta B_{2}}=-\dot{p}_{B_{2}},
\end{array}
$$

are identical to the operator equations of motion obtained from the total trace Lagrangian. So we have achieved a consistent Hamiltonian dynamics. The generalized Poisson bracket of Eq. (6a), in axial gauge, now contains variational derivatives only with respect to the gluon variables $B_{1,2}$ and $p_{B_{1,2}}$. We have not verified the Poincaré generator algebra, but just as with the verification of the Hamiltonian equations of 
motion which we have described, this should be a straightforward analog of the conventional Yang-Mills axial gauge calculation.

At no point in the discussion have canonical commutation relations been used to get the operator equations of motion. They have been replaced in total trace dynamics by the constraints

$$
0=\mathcal{J}_{0}+\sum_{\ell=1}^{3} \hat{D}_{\ell} p_{B_{\ell}}, \quad 0= \pm \mathcal{J}_{0}^{\prime}+\sum_{\ell=1}^{3} \hat{D}_{\ell}^{\prime} p_{B_{\ell}^{\prime}},
$$

and are a quaternionic field theory generalization, for independent left and right gaugings, of the constraints of Eq. (14b) of Sec. 2. We conjecture that any operator realization of Eq. (54) gives, via the total trace Hamiltonian formalism of Eqs. (47a)-(53), a consistent quaternionic field dynamics.

\section{Discussion}

In the preceding three sections, we have presented a generalization of standard quantum mechanics, providing a framework within which one can formulate quaternionic quantum field theories. We suggest in the final chapter of [1] that such theories may play a role in physics between the GUTS scale and the Planck mass, possibly providing a dynamics for preon models of quarks and leptons, and that the observed complex quantum field theories associated with the standard model and its grand unification are effective theories describing the asymptotic dynamics of the underlying quaternionic fields.

These speculations aside, the concepts of total trace Lagrangian and Hamiltonian dynamics, and operator gauge invariance, provide rich new possibilities for the formulation and study of quantum field systems. Among the many open questions which remain are: (1) Can the generalized bracket of Eq. (6a) be proved to satisfy a Jacobi identity? (2) What is the analog of the Dirac theory of constrained systems for operator gauge invariant systems? (3) What is the analog of BRST theory for such systems? (4) For the gauge models discussed in axial gauge in Sec. 4, what form does the total trace Hamiltonian take in non-canonical gauges, such as transverse gauge? (5) Does total trace dynamics, in the quaternionic case, correspond to a unitary time development, or is this only a property of complex quantum mechanics? (6) Can one usefully characterize the solutions of the operator constraints of Eq. (54)? (7) Given two scalar or fermion quaternionic operator fields, is there a criterion for determining whether they are related by a bi-unitary operator gauge 
transformation? (8) Can one find a functional integration form of total trace dynamics, analogous to the Feynman path integral in complex quantum field theory? (9) More generally, what are useful calculational techniques for the theories formulated in this paper?

\section{Acknowledgement}

I wish to thank many colleagues, cited in full in [1], for helpful conversations and correspondence dealing with quaternionic quantum mechanics, quantum field theory, and related issues. This work was supported in part by the Department of Energy under Grant \#DE-FG02-90ER40542. 


\section{References}

[ 1] S.L. Adler, Quaternionic quantum mechanics and quantum fields (Oxford University Press, Oxford, 1994).

[ 2] E. Witten, J. Diff. Geom. 17 (1982) 661.

[ 3] S.L. Adler, Phys. Lett. 86B (1979) 203.

[ 4] S.L. Adler, Phys. Rev. D21 (1980) 550.

[ 5] S.L. Adler, Phys. Rev. D21 (1980) 2903.

[ 6] D. Finkelstein, J.M. Jauch and D. Speiser, Notes on quaternion quantum mechanics (1959), published in C. Hooker, ed., Logico-algebraic approach to quantum mechanics II (Reidel, Dordrecht, 1979).

[ 7] G.W. Mackey, Weyl's program and modern physics (1987), in K. Bleuler and M. Werner, eds., Differential geometric methods in theoretical physics (Kluwer Academic Publishers, Dordrecht, 1988).

[ 8] G.W. Mackey, The axiomatics of particle interactions (1992), talk at the Castiglioncello conference, Int. J. Theoret. Phys. (in press).

[ 9] A. Connes, Publ. Math. IHES 62 (1983) 44.

[ 10] A. Connes, Essay on physics and non-commutative geometry, in D. Quillen, G. Segal and S. Tsau, eds., The interface of mathematics and particle physics (Clarendon Press, Oxford, 1990).

[ 11] D. Finkelstein, J.M. Jauch, S. Schiminovich and D. Speiser, J. Math. Phys. 4 (1963) 788.

[ 12] L.P. Horwitz and L.C. Biedenharn, Ann. Phys. 157 (1984) 432.

[ 13] O. Teichmüller, J. Reine Angew. Math. 174 (1935) 73.

[ 14] D. Finkelstein, J.M. Jauch and D. Speiser, J. Math. Phys. 4 (1963) 136.

[ 15] A. Razon and L.P. Horwitz, Acta Applicandae Mathematicae 24 (1991) 141.

[ 16] A. Razon and L.P. Horwitz, Acta Applicandae Mathematica 24 (1991) 179.

[ 17] A. Razon and L.P. Horwitz, J. Math. Phys. 33 (1992) 3098.

[ 18] P. Cvitanovíc, R.J. Gonsalves and D.E. Neville, Phys. Rev. D18 (1978) 3881.

[ 19] S.C. Lee, Phys. Rev. D20 (1979) 1951.

[ 20] S.C. Lee, Phys. Rev. D21 (1980) 466. 
[ 21] K.A. Milton, W.F. Palmer and S.S. Pinsky, Phys. Rev. D25 (1982) 1718.

[ 22] S.L. Adler, Phys. Rev. D17 (1978) 3212.

[ 23] E. Witten, Phys. Lett. 117B (1982) 324.

[ 24] S. Weinberg, Gravitation and cosmology (John Wiley, New York, 1972) Sec. 12.2.

[ 25] S. Ferrara and B. Zumino, Nucl. Phys. B79 (1974) 413.

[ 26] A. Salam and J. Strathdee, Phys. Lett. 51B (1974) 353.

[ 27] R.L. Arnowitt and S.I. Fickler, Phys. Rev. 127 (1962) 1821.

[ 28] A. Hanson, T. Regge and C. Teitelboim, Constrained Hamiltonian systems (Acad. Naz. dei Lincei, Rome, 1976). 